\title{
Spin-wave condensation and quantum melting of long-range antiferromagnetic order in $t-J$ model.
}

\author{
O. P. Sushkov ${ }^{a}$ \\ School of Physics, The University of New South Wales Sydney 2052, Australia
}

\begin{abstract}
Ground state wave function of two-dimensional $t-J$ model is found at doping close to half filling. It is shown that the condensation of Cooper pairs (superconducting pairing of mobile holes) and the condensation of spin-waves into spin-liquid state are closely connected. The effective spectrum of $S=1$ excitations, spin-wave gap pseudo-gap $\Delta_{M}$, magnetic correlation length $\xi_{M}$, and static magnetic formfactor $S_{M}(\mathbf{q})$ are calculated.
\end{abstract}

PACS numbers: 71.27.+a, 74.20.Hi, 75.50.Ee

\section{INTRODUCTION}

In the recent papers [1,2] in the framework of $t-J$ model we have demonstrated very strong $d$-wave pairing between dressed quasiholes induced by spin-wave exchange. The pairing gives critical temperature in a reasonable agreement with experimental data for copper oxide superconductors. In general terms our approach follows the studies, of Monthoux, Pines, Scarlapino, Schriffer, and others, supporting idea of the magnetic fluctuations mechanism of pairing [3] 8]. For calculations we have used BCS-like approximation for dressed quasiholes. A similar approach to the pairing of dressed quasiholes has been used by Dagotto, Nazarenko, and Moreo [9]. The important difference is that in our work [1,2] the hole-hole interaction is derived from the parameters of the t-J model, while in [9] it is introduced $a d$ hoc with magnitude adjusted to fit experimental data.

We consider the holes as well as spin-waves moving in the background with long-range antiferromagnetic (AF) order. It means that we assume that the AF order is preserved at distances smaller than inverse Fermi momentum $r \leq 1 / p_{F}$. It is widely believed that this assumption is correct and present work confirms this point. It is also widely believed that correct spin structure of the ground state should be of the type of Anderson spin-liquid state [10]. For $t-J$ model the instability of long-range AF order together with normal-hole Fermi liquid was probably first pointed out by Shraiman and Siggia [11. Later it has been demonstrated in numerous works (e.g. see Refs. [12 16]). It is due to the strong interaction of spin-waves with mobile holes. However, a structure of the ground state as well as a spectrum of excitations was not understood, and spin-liquid state was not derived.

Now we understand that we have to consider a hole-Fermi-liquid with superconducting pairing which interacts with long-range fluctuations of AF ordering. Physical difference of the paired Fermi-liquid from normal one is stiffness of the former. In the present work we demonstrate that the long-range AF order is also destroyed, but pairing is preserved. We 
derive explicitly the spin-liquid state of the system. We show that selfconsistent translation invariant spin-liquid solution exists at doping $\delta \geq \delta_{c} \approx 3-4 \%$. Using this solution we calculate spin-wave pseudo-gap $\Delta_{M}$, magnetic correlation length $\xi_{M}$ and static magnetic formfactor $S(\mathbf{q})$. In general terms the results which we derive here in the framework of $t-J$ model are very close to the picture of magnetic state discussed in the works of Chubukov, Pines, Sachdev, Sokol, and Ye [17 20].

The paper has the following structure. In Sec.II we present the effective Hamiltonian of $t-J$ model and discuss pairing of holes. In Sec.III we formulate the idea of our approach presenting the simplified calculation of spin-wave Green's function. Sec.IV presents calculation of spin-wave polarization operator. In Sec.V spin-wave Green's function is calculated. We also discuss the pseudo-gap in spin-wave spectrum. Sec.VI presents calculations of magnetic correlation length $\xi_{M}$ and static magnetic formfactor $S_{M}(\mathbf{q})$. Finally our conclusions are given in Sec. VII.

\section{EFFECTIVE HAMILTONIAN AND PAIRING OF DRESSED HOLES}

The $t-J$ model is defined by the Hamiltonian

$$
H=H_{t}+H_{J}=-t \sum_{<n m>\sigma}\left(d_{n \sigma}^{\dagger} d_{m \sigma}+\text { H.c. }\right)+J \sum_{<n m>} \mathbf{S}_{n} \cdot \mathbf{S}_{m}
$$

where $d_{n \sigma}^{\dagger}$ is the creation operator of a hole with spin $\sigma(\sigma=\uparrow, \downarrow)$ at site $n$ on a twodimensional square lattice. The $d_{n \sigma}^{\dagger}$ operators act in the Hilbert space with no double electron occupancy. The spin operator is $\mathbf{S}_{n}=\frac{1}{2} d_{n \alpha}^{\dagger} \boldsymbol{\sigma}_{\alpha \beta} d_{n \beta} .<n m>$ are the nearest-neighbour sites on the lattice. Below we set $J$ as well as lattice spacing equal to unity.

At half-filling (one hole per site) $t$ - $J$ model is equivalent to the Heisenberg AF model [21,22] which has long-range AF order in the ground state [23,24]. Let us denote the wave function of this ground state by $|0\rangle$. This is undoped system. We consider the doped system basing on the ground state of undoped one. In spite of destruction of the long-range AF order it is convenient to use $|0\rangle$ and corresponding quasiparticle excitations as a basis set in the problem with doping. The effective Hamiltonian for $t-J$ model in terms of these quasiparticles was derived in the papers 25. 27.

$$
H_{e f f}=\sum_{\mathbf{k} \sigma} \epsilon_{\mathbf{k}} h_{\mathbf{k} \sigma}^{\dagger} h_{\mathbf{k} \sigma}+\sum_{\mathbf{q}} \omega_{\mathbf{q}}\left(\alpha_{\mathbf{q}}^{\dagger} \alpha_{\mathbf{q}}+\beta_{\mathbf{q}}^{\dagger} \beta_{\mathbf{q}}\right)+H_{h, s w}+H_{h h}
$$

It is expressed in terms of usual spin-waves on AF background $\alpha_{\mathbf{q}}, \beta_{\mathbf{q}}$ (see e.g. review [28]), and composite hole operators $h_{\mathbf{k} \sigma}(\sigma= \pm 1 / 2)$. The summations over $\mathbf{k}$ and $\mathbf{q}$ are restricted inside the Brillouin zone of one sublattice where $\gamma_{\mathbf{q}}=\frac{1}{2}\left(\cos q_{x}+\cos q_{y}\right) \geq 0$. Spin-wave dispersion is

$$
\omega_{\mathbf{q}}=2 \sqrt{1-\gamma_{\mathbf{q}}^{2}}, \quad \omega_{\mathbf{q}} \approx \sqrt{2}|\mathbf{q}|, \text { at } q \ll 1
$$

Single hole properties are well established (for a review see Ref. [29]). Wave function of a single hole can be represented as $\psi_{\mathbf{k} \sigma}=h_{\mathbf{k} \sigma}^{\dagger}|0\rangle$. At large $t$ the composite hole operator $h_{\mathbf{k} \sigma}^{\dagger}$ has complex structure. For example at $t / J=3$ the weight of bare hole in $\psi_{\mathbf{k} \sigma}$ is about $25 \%$, the weight of configurations "bare hole +1 magnon" is $\sim 50 \%$, and of configurations "bare hole +2 or more magnons" $\sim 25 \%$. Dressed hole is a normal fermion. Hole energy $\epsilon_{\mathbf{k}}$ has minima at $\mathbf{k}=\mathbf{k}_{0}$, where $\mathbf{k}_{0}=( \pm \pi / 2, \pm \pi / 2)$. For $t \leq 5$ the dispersion can be well approximated by the expression [30] 


$$
\epsilon_{\mathbf{k}} \approx E_{0}+2-\sqrt{0.66^{2}+4.56 t^{2}-2.8 t^{2} \gamma_{\mathbf{k}}^{2}}+\frac{1}{4} \beta_{2}\left(\cos k_{x}-\cos k_{y}\right)^{2} .
$$

The decimals in this formula are some combinations of the Heisenberg model spin correlators. Constant $E_{0}$ defines reference level for the energy. To find $\epsilon_{\mathbf{k}}$ with respect to undoped system one has to set $E_{0}=0$. However for present work it is convenient to set $\epsilon_{\mathbf{k}_{0}}=0$, and therefore $E_{0}=\sqrt{0.66^{2}+4.56 t^{2}}-2$. The coefficient $\beta_{2}$ is small and therefore the dispersion is almost degenerate along the face of the magnetic Brillouin zone $\gamma_{\mathbf{k}}=0$. According to Refs. [31, 32, $\beta_{2} \approx 0.1 \cdot t$ at $t \geq 0.33$. To avoid misunderstanding we note that formula (4) certainly is not valid for very large $t$ where the hole band width is saturated at the value of the order of unity and does not increase with $t$. However physically we are interested in $t \approx 3$ (e.g., see Refs. [33 35]) where (4) works well. Near the band minima $\mathbf{k}_{0}$ the dispersion (4) can be presented in the usual quadratic form

$$
\epsilon_{\mathbf{p}} \approx \frac{1}{2} \beta_{1} p_{1}^{2}+\frac{1}{2} \beta_{2} p_{2}^{2}, \quad \beta_{2} \ll \beta_{1},
$$

where $p_{1}\left(p_{2}\right)$ is the projection of $\mathbf{k}-\mathbf{k}_{0}$ on the direction orthogonal (parallel) to the face of the magnetic Brillouin zone (Fig.1). From Eq.(四) for $t \gg 0.33$ we find $\beta_{1} \approx 0.65 t$, hence the mass anisotropy is $\beta_{1} / \beta_{2} \approx 7$.

The interaction of a composite hole with spin waves is of the form (see, e.g., Refs. [31, 36, 250)

$$
\begin{aligned}
& H_{h, s w}=\sum_{\mathbf{k}, \mathbf{q}} g_{\mathbf{k}, \mathbf{q}}\left(h_{\mathbf{k}+\mathbf{q} \downarrow}^{\dagger} h_{\mathbf{k} \uparrow} \alpha_{\mathbf{q}}+h_{\mathbf{k}+\mathbf{q} \uparrow}^{\dagger} h_{\mathbf{k} \downarrow} \beta_{\mathbf{q}}+\text { H.c. }\right), \\
& g_{\mathbf{k}, \mathbf{q}}=2 \sqrt{2} f\left(\gamma_{\mathbf{k}} U_{\mathbf{q}}+\gamma_{\mathbf{k}+\mathbf{q}} V_{\mathbf{q}}\right),
\end{aligned}
$$

where $U_{\mathbf{q}}=\sqrt{\frac{1}{\omega_{\mathbf{q}}}+\frac{1}{2}}$ and $V_{\mathbf{q}}=-\operatorname{sign}\left(\gamma_{\mathbf{q}}\right) \sqrt{\frac{1}{\omega_{\mathbf{q}}}-\frac{1}{2}}$ are the parameters of Bogoliubov transformation diagonalizing spin-wave Hamiltonian. The hole spin-wave coupling constant $f$ is a function of $t$ evaluated in the work [25]. For large $t$ the coupling constant is $t$-independent $f \approx 2$. Let us stress that even for $t>J$ the quasihole-spin-wave interaction (6) has the same form as for $t \ll J$ (i.e. as for bare hole operators) with an added renormalization factor (of the order $J / t$ for $t \gg J)$. This remarkable property of the $t-J$ model is due to the absence of single loop correction to the hole-spin-wave vertex. It was first stated implicitly by Kane, Lee, and Read [37]. In Refs. [31,36, 25] it was explicitly demonstrated that the vertex corrections with different kinematic structure are of the order of few percent at $t / J \approx 3$. There is also some $q$-dependence of the coupling constant $f$. For example $f(q=\pi) \approx 1.15 f(q=0)$ at $t / J=3$ (see Refs. 27, 2]). However this dependence is weak, it is practically beyond the accuracy of the calculation of the renormalized value of $f$. Therefore we neglect this dependence.

Finally there is contact hole-hole interaction $H_{h h}$ in the effective Hamiltonian (2). $H_{h h}$ is discussed in details in the Refs. [26,27,2]. It is proportional to some function $A(t)$. For small $t$ this function approaches the value -0.25 , which gives the well known hole-hole attraction induced by the reduction of the number of missing antiferromagnetic links. However for realistic superconductors $t \approx 3$ (see e.g.Refs. 33 35]). Surprisingly function $A(t)$ vanishes exactly at $t \approx 3$, and it means that the mechanism of contact hole-hole attraction is switched off. In contrast the spin-wave exchange mechanism $H_{h, s w}$ is negligible for small $t$ where $f \sim t$, and it is most important at large $t$ where $f$ approaches 2 . We are interested in "physical" values of $t: t \approx 3$. Therefore in the present work we neglect contact interaction $\left(H_{h h}=0\right)$ and consider only the hole-spin-wave interaction at $t=3$. Corresponding value of $f$ according to [25] is $f=1.8$. 
The interaction which gives hole-hole superconducting pairing arises from spin-wave exchange (Fig.2). From (2) we find

$$
V_{\mathbf{k}, \mathbf{k}^{\prime}}=-2 \frac{g_{\mathbf{k}, \mathbf{q}} g_{\mathbf{k}^{\prime},-\mathbf{q}}}{-\omega_{\mathbf{q}}-E_{\mathbf{k}}-E_{\mathbf{k}^{\prime}}}
$$

where $\mathbf{q}=\mathbf{k}+\mathbf{k}^{\prime}$, and $E_{\mathbf{k}}$ is the energy of quasihole excitation above the BCS ground state (see below). We consider the interaction in static approximation. Justification of this approximation is as follows. At small hole concentration $\delta \ll 1$, the holes are localized in momentum space in the vicinity of the minima of the band $\mathbf{k}_{0}=( \pm \pi / 2, \pm \pi / 2)$ and Fermi surface consists of ellipses. The Fermi energy and Fermi momentum of non-interacting holes are

$$
\epsilon_{F} \approx \frac{1}{2} \pi\left(\beta_{1} \beta_{2}\right)^{1 / 2} \delta, \quad p_{F} \approx \sqrt{p_{1 F} p_{2 F}} \approx(\pi \delta)^{1 / 2}
$$

The Fermi momentum $p_{F}$ is measured from the center of corresponding ellipse. In pairing, the exchange of spin-waves with typical momenta $\pi>q>p_{F}$ is the most important (see also Refs. [1,22,27]). The energy of such spin wave is much higher than the typical energy of a Cooper pair

$$
\omega_{q} \sim q>p_{F} \sim(\pi \delta)^{1 / 2} \gg \epsilon_{F} \sim\left(\beta_{1} \beta_{2}\right)^{1 / 2} \delta .
$$

This justifies the static approximation (7) for hole-hole interaction (see also comment at end of section V). This is much different from usual phonon induced pairing where Debye's frequency is much lower than the Fermi energy.

With trial function of ground state

$$
|\Psi\rangle=\prod_{\mathbf{k}}\left(u_{\mathbf{k}}+v_{\mathbf{k}} h_{\mathbf{k} \uparrow}^{\dagger} h_{-\mathbf{k} \downarrow}^{\dagger}\right)|0\rangle .
$$

we get conventional BCS equation for superconducting gap $\Delta_{\mathbf{k}}$

$$
\begin{aligned}
& \Delta_{\mathbf{k}}=-\frac{1}{2} \sum_{\mathbf{k}^{\prime}} V_{\mathbf{k k}^{\prime}} \frac{\Delta_{\mathbf{k}^{\prime}}}{E_{\mathbf{k}^{\prime}}} \tanh \frac{E_{\mathbf{k}^{\prime}}}{2 T} \\
& E_{\mathbf{k}}=\sqrt{\xi_{\mathbf{k}}^{2}+\Delta_{\mathbf{k}}^{2}} \\
& u_{\mathbf{k}}=\sqrt{\frac{1}{2}\left(1+\xi_{\mathbf{k}} / E_{\mathbf{k}}\right)} \\
& v_{\mathbf{k}}=\operatorname{sign} \Delta_{\mathbf{k}} \cdot \sqrt{\frac{1}{2}\left(1-\xi_{\mathbf{k}} / E_{\mathbf{k}}\right)}
\end{aligned}
$$

where $\xi_{\mathbf{k}}=\epsilon_{\mathbf{k}}-\mu$, and $\mu$ is chemical potential fixed by equation

$$
\delta=2 \sum_{\mathbf{k}}\left(v_{\mathbf{k}}^{2}+\frac{u_{\mathbf{k}}^{2}-v_{\mathbf{k}}^{2}}{e^{E_{\mathbf{k}} / T}+1}\right)
$$

All details of approximate analytical and exact numerical solutions of equation (11) are discussed in our papers [1,2]. This equation has an infinite set of solutions of different symmetries with strongest pairing in the d-wave (four nodes of the gap at the face of magnetic Brillouin zone). For further discussion we need only the results for d-wave. All numerical values are calculated at $t=3, \beta_{1} / \beta_{2}=7$, and $f=1$.8. In Table I we present for different values of hole concentration 1)Fermi energy $\epsilon_{F}, 2$ )chemical potential at zero temperature $\mu(0), 3)$ maximum value of the gap in Brillouin zone at zero temperature $\left.\Delta_{\max }(0), 4\right)$ maximum value of the gap at Fermi surface at zero temperature $\left.\Delta_{F \max }(0), 5\right)$ chemical potential at critical temperature $\left.\mu\left(T_{c}\right), 6\right)$ Critical temperature $T_{c}$. In Fig. 1 we give the map of a single hole mean occupation number $v_{\mathbf{k}}^{2}$ at $\delta=0.1$ and zero temperature. 


\section{MODIFIED SPIN-WAVE THEORY AND QUANTUM MELTING OF LONG-RANGE ANTIFERROMAGNETIC ORDER}

It is well known that in the long wave-length limit Heisenberg model is equivalent to the nonlinear $\sigma$-model (e.g., see review paper [28]). Therefore usual field theory crossing symmetry is valid. The same is valid for $t-J$ model. Technically it is evident from Eq.(6): at $q \ll 1$ the vertex $g_{\mathbf{k}, \mathbf{q}} \approx g_{\mathbf{k}-\mathbf{q}, \mathbf{q}}$. This means that instead of considering a set of Green's functions $\left\langle\alpha_{\mathbf{k}} \alpha_{\mathbf{k}}^{\dagger}\right\rangle,\left\langle\alpha_{\mathbf{k}} \beta_{-\mathbf{k}}\right\rangle \ldots$, one can introduce one combined Green's function of vector excitation

$$
D(\omega, \mathbf{q})=\frac{2 \omega_{\mathbf{q}}}{\omega^{2}-\omega_{q}^{2}-2 \omega_{\mathbf{q}} \Pi(\omega, \mathbf{q})}
$$

where $\Pi(\omega, \mathbf{q})$ is mobile holes polarization operator. For stability of the system the condition

$$
\omega_{q}+2 \Pi(0, \mathbf{q})>0
$$

should be fulfilled. Otherwise the Green's function (13) would possess poles with imaginary $\omega$. It was demonstrated in the papers [11 16] that with $\Pi(0, \mathbf{q})$ calculated in the normal hole Fermi liquid approximation the condition (14) is violated. It means the instability of longrange AF order. Now we want to: 1)Take into account strong hole-hole pairing, 2)Formulate the approach for description of state without long-range AF order. In the present section we discuss only point 2).

For elucidation of physical meaning of our approach it is convenient to use Hamiltonian technique instead of the Feynman one. Due to the interaction with mobile holes the wave function of the renormalized spin-wave corresponding to the Green's function (13) is a combination of $\alpha_{\mathbf{q}}^{\dagger}$ and $\beta_{-\mathbf{q}}$. To find this wave function let us write down the effective spin-wave Hamiltonian.

$$
H_{s w}=\sum_{\mathbf{q}}\left(\left(\omega_{\mathbf{q}}+\Pi(\omega, \mathbf{q})\right)\left(\alpha_{\mathbf{q}}^{\dagger} \alpha_{\mathbf{q}}+\beta_{\mathbf{q}}^{\dagger} \beta_{\mathbf{q}}\right)-\Pi(\omega, \mathbf{q})\left(\alpha_{\mathbf{q}} \beta_{-\mathbf{q}}+\alpha_{\mathbf{q}}^{\dagger} \beta_{-\mathbf{q}}^{\dagger}\right)\right) .
$$

The term proportional to $\omega_{\mathbf{q}}$ comes from the "bare" Hamiltonian (2). First "П-term" comes from the diagram Fig.3a where one spin-wave is annihilated and the other is created. Second "П-term" comes from diagrams Fig.3bc where two spin-waves are annihilated or created. Let us note that spin-waves have definite values of $S_{z}: \alpha_{\mathbf{q}}^{\dagger}$ has $S_{z}=-1$ and $\beta_{-\mathbf{q}}$ has $S_{z}=+1$. Therefore they can appear only in combinations presented in (15). One can certainly prove this explicitly using (6) and calculating the polarization operator. In the second "П-term" the spin-waves have the opposite momenta. At $q \ll 1$ the vertex $g_{\mathbf{k}, \mathbf{q}}$ is proportional to the momentum $q$. Just for this reason second "П-term" has different sign in comparison with first one. Diagonalization of the Hamiltonian (15) by the Bogoliubov transformation gives the spectrum of Bose excitations in the system

$$
\Omega_{\mathbf{q}}^{2}=\omega_{\mathbf{q}}^{2}+2 \omega_{\mathbf{q}} \Pi\left(\Omega_{\mathbf{q}}, \mathbf{q}\right) .
$$

This is exactly the equation for the poles of the Green's function (13). So the Hamiltonian approach reproduces conventional result of the Feynman technique. Account of $\omega$-dependence of the polarization operator in Hamiltonian technique is rather cumbersome. Therefore in the present section we neglect it: $\Pi\left(\Omega_{\mathbf{q}}, \mathbf{q}\right) \approx \Pi(0, \mathbf{q})$. As usual Bogoliubov transformation gives the ground state of the form

$$
|g s\rangle \propto \exp \left(\sum_{\mathbf{q}} c_{\mathbf{q}} \alpha_{\mathbf{q}}^{\dagger} \beta_{-\mathbf{q}}^{\dagger}\right)|0\rangle
$$


with some coefficients $c_{\mathbf{q}}$. This is the condensate of spin-waves.

We started from Neel ground state $|0\rangle$ with two sublattices $u$-up and $d$-down. The difference in magnetization of the two sublattices is of the form (see e.g. Ref. [38])

$$
\frac{1}{2}\left(S_{u}^{z}-S_{d}^{z}\right)=0.303-2 \sum_{\mathbf{q}} \frac{1}{\omega_{\mathbf{q}}}\left(\alpha_{\mathbf{q}}^{\dagger} \alpha_{\mathbf{q}}+\beta_{\mathbf{q}}^{\dagger} \beta_{\mathbf{q}}-\gamma_{\mathbf{q}}\left(\alpha_{\mathbf{q}} \beta_{-\mathbf{q}}+\alpha_{\mathbf{q}}^{\dagger} \beta_{-\mathbf{q}}^{\dagger}\right)\right) .
$$

Using parameters of transformation which diagonalizes the Hamiltonian (15) one can easily calculate the renormalized magnetization

$$
\delta S_{z}=\left\langle g s\left|\frac{1}{2}\left(S_{u}^{z}-S_{d}^{z}\right)\right| g s\right\rangle=0.303-2 \int\left(\frac{1}{\Omega_{\mathbf{q}}}-\frac{1}{\omega_{\mathbf{q}}}\right) \frac{d^{2} \mathbf{q}}{(2 \pi)^{2}},
$$

where $\Omega_{\mathbf{q}}=\sqrt{\omega_{\mathbf{q}}^{2}+2 \omega_{\mathbf{q}} \Pi(0, \mathbf{q})}$.

In the above discussion we assumed that condition (14) is fulfilled and hence $\Omega_{\mathbf{q}}$ is real. Let us now increase polarization operator $(\Pi(0, \mathbf{q}) \rightarrow x \cdot \Pi(0, \mathbf{q}), x>1)$ approaching $\Omega_{\mathbf{q}}$ to zero. There are two possibilities: 1) $\Omega_{\mathbf{q}}$ vanishes at some values of $\mathbf{q}$, but $\delta S_{z}$ remains finite positive because of the convergence of the integral in (19). 2) In approaching $\Omega_{\mathbf{q}}$ to zero $\delta S_{z}$ vanishes and then becomes negative. The choice between these two scenario depends on $\mathbf{q}$-dependence of $\Pi(0, \mathbf{q})$. We will demonstrate below that for paired hole Fermi liquid at $\delta>\delta_{c}$ second scenario is realized. Therefore let us discuss this situation.

Vanishing of the magnetization $\delta S_{z}$ means that there is a lot of spin-waves in the condensate (17) and we have to take into account their nonlinear interaction. We can not do it exactly. Fortunately, there is approximate way. We can apply the modified spin-wave theory suggested by Takahashi for the Heisenberg model at nonzero temperature [38. Following Takahashi let us impose the condition that the sublattice magnetization vanishes in the quantum spin-liquid state

$$
\left\langle g s\left|\frac{1}{2}\left(S_{u}^{z}-S_{d}^{z}\right)\right| g s\right\rangle=0 .
$$

To find the ground state with this condition we have to diagonalize

$$
H_{\nu}=H_{s w}-\frac{1}{8} \nu^{2}\left(S_{u}^{z}-S_{d}^{z}\right)
$$

where $\frac{1}{8} \nu^{2}$ is Lagrange multiplier, $H_{s w}$ is given by (15) and $\left(S_{u}^{z}-S_{d}^{z}\right)$ by (18). Simple calculation shows that instead of (16) we get a spectrum of excitations with a gap

$$
\Omega_{\nu \mathbf{q}}=\sqrt{\Omega_{\mathbf{q}}^{2}+\nu^{2}}
$$

The average value of the magnetization is given by the formula (19) with $\Omega_{\mathbf{q}}$ replaced by $\Omega_{\nu \mathbf{q}}$. The gap $\nu$ should be found after substitution of this formula into condition (20). Let us stress that this condition reflects strong nonlinearity of the spin-wave theory. In essence it gives an effective cutoff of unphysical states in the Dyson-Maleev approach. This question is discussed in the paper [39] where the modified spin-wave theory is applied to the description of the transition from $\mathrm{AF}$ state to spin-liquid state in $J_{1}-J_{2}$ model.

In the conclusion of present section we would like to note that realization of the first scenario (finite positive magnetization $\delta S_{z}$ at vanishing of $\Omega_{\mathbf{q}}$ at some values of $\mathbf{q}$ ) would mean instability with respect to decay to spiral state. It happens at $\delta<\delta_{c}$. 


\section{SPIN-WAVE GREEN'S FUNCTIONS AND POLARIZATION OPERATORS}

We are interested in hole concentration $\delta \sim 0.15$. Corresponding value of $q$ is $q \sim p_{F} \sim 1$. Therefore, for practical calculations $\sigma$-model long-wave-length approximation (13) is not enough. The technique which overcomes this problem was developed by Igarashi and Fulde [40], see also the paper by Khaliullin and Horsch [41. Following these works let us introduce the Green's functions for spin-waves

$$
\begin{aligned}
& D_{\alpha \alpha}(t, \mathbf{q})=-i\left\langle T\left[\alpha_{\mathbf{q}}(t) \alpha_{\mathbf{q}}^{\dagger}(0)\right]\right\rangle, \\
& D_{\alpha \beta}(t, \mathbf{q})=-i\left\langle T\left[\alpha_{\mathbf{q}}(t) \beta_{-\mathbf{q}}(0)\right]\right\rangle \\
& D_{\beta \alpha}(t, \mathbf{q})=-i\left\langle T\left[\beta_{-\mathbf{q}}^{\dagger}(t) \alpha_{\mathbf{q}}^{\dagger}(0)\right]\right\rangle \\
& D_{\beta \beta}(t, \mathbf{q})=-i\left\langle T\left[\beta_{-\mathbf{q}}^{\dagger}(t) \beta_{-\mathbf{q}}(0)\right]\right\rangle,
\end{aligned}
$$

where $T$ is the time-ordering operator, and $\langle\ldots\rangle$ represents an average over the exact ground state. In zero approximation in the interaction with mobile holes the spin-wave Green's functions in $\omega$-q representation are given by 40

$$
\begin{aligned}
& D_{\alpha \alpha}^{0}(\omega, \mathbf{q})=\left(\omega-\omega_{\mathbf{q}}+i 0\right)^{-1} \\
& D_{\alpha \beta}^{0}(\omega, \mathbf{q})=D_{\beta \alpha}^{0}(\omega, \mathbf{q})=0 \\
& D_{\beta \beta}^{0}(\omega, \mathbf{q})=\left(-\omega-\omega_{\mathbf{q}}+i 0\right)^{-1}
\end{aligned}
$$

Polarization operator in this technique is also a matrix. So there are $P_{\alpha \alpha}(\omega, \mathbf{q}), P_{\alpha \beta}(\omega, \mathbf{q})$, $P_{\beta \alpha}(\omega, \mathbf{q})$, and $P_{\beta \beta}(\omega, \mathbf{q})$ components.

In one loop approximation polarization operator is given by the diagrams presented in Fig.4. where the normal as well as the anomalous hole Green's function is taken into account. First of all let us discuss contributions of the "coherent" and "incoherent" parts of the hole Green's function into the polarization operator. The diagrams in Fig.4 are represented in terms of the dressed quasiholes $h_{\mathbf{k}}$ which involve in the effective Hamiltonian (2). However one can ask the question: what is the accuracy of this approximation and what happens if we substitute the Green's function of bare hole $d_{n \sigma}$ into polarization operator? The Green's function of bare hole is of the form

$$
G(\epsilon, \mathbf{k})=\frac{Z}{\epsilon-\epsilon_{\mathbf{k}}+i 0}+G_{i n c o h} .
$$

where $Z$ is quasiparticle residue. If we use bare hole Green's function we also have to use bare hole-spin-wave coupling constant $f_{\text {bare }}=2 t$ in the vertex $g_{\mathbf{k}, \mathbf{q}}$ (6). Substitution of the pole (coherent) part of (25) into polarization operator gives combination $f_{\text {bare }} Z$, but this is exactly the effective coupling constant $f$ [25]. Thus, the effective theory with Hamiltonian (2) is equivalent to the account of the coherent (pole) part of the bare hole Green's function (25). According to condition (14) the most important characteristic is the polarization operator at zero frequency. If we neglect the pairing of quasiholes we can easily calculate its value in normal Fermi-liquid approximation [16])

$$
P_{\alpha \alpha}(0, \mathbf{q})=\Pi(0, \mathbf{q}) \approx-\frac{\sqrt{2} f^{2}}{\pi \sqrt{\beta_{1} \beta_{2}}} q, \quad \text { at } \quad q \ll p_{F} .
$$

This is coherent contribution. It is independent of the hole concentration! Incoherent part of the polarization operator which comes from $G_{i n c o h}$ in (25)is proportional to hole concentration $\delta$. Therefore it is negligible at $\delta \ll 1$. This conclusion agrees with that of the Ref. 42]. It is interesting that incoherent contribution can be also calculated analytically 434] 


$$
P_{\text {incoh }}(0, \mathbf{q}) \approx-\frac{f^{4}}{4 \sqrt{2} \pi} \delta \ln \frac{1}{\delta} \cdot q .
$$

At any reasonable $\delta$ the incoherent part $P_{\text {incoh }}(0, \mathbf{q})$ does not exceed $10 \%$ of $P_{\text {coh }}(0, \mathbf{q})$. Therefore further we neglect $P_{\text {incoh }}$ and use effective theory with the Hamiltonian (2) which is equivalent to the account of only coherent part in the hole Green's function (25).

Due to the symmetry of Green's function it is convenient to introduce following notations

$$
\begin{aligned}
& P_{\beta \beta}(-\omega, \mathbf{q})=P_{\alpha \alpha}(\omega, \mathbf{q})=\Pi(\omega, \mathbf{q}) \\
& P_{\alpha \beta}(\omega, \mathbf{q})=P_{\beta \alpha}(\omega, \mathbf{q})=\bar{\Pi}(\omega, \mathbf{q}) .
\end{aligned}
$$

Calculation of the polarization operator Fig.4 in single loop approximation is straightforward. Account of both normal and anomalous hole Green's functions gives

$$
\begin{aligned}
\Pi^{(1)}(\omega, \mathbf{q})= & \sum_{\mathbf{k}}\left[v_{\mathbf{k}}^{2} u_{\mathbf{k}+\mathbf{q}}^{2}\left(\frac{g_{\mathbf{k}, \mathbf{q}}^{2}}{\omega-E_{\mathbf{k}}-E_{\mathbf{k}+\mathbf{q}}}+\frac{g_{\mathbf{k}+\mathbf{q},-\mathbf{q}}^{2}}{-\omega-E_{\mathbf{k}}-E_{\mathbf{k}+\mathbf{q}}}\right)-\right. \\
& \left.-u_{\mathbf{k}} v_{\mathbf{k}} u_{-\mathbf{k}-\mathbf{q}} v_{-\mathbf{k}-\mathbf{q}} g_{\mathbf{k}, \mathbf{q}} g_{\mathbf{k}+\mathbf{q},-\mathbf{q}}\left(\frac{1}{\omega-E_{\mathbf{k}}-E_{\mathbf{k}+\mathbf{q}}}+\frac{1}{-\omega-E_{\mathbf{k}}-E_{\mathbf{k}+\mathbf{q}}}\right)\right], \\
\bar{\Pi}^{(1)}(\omega, \mathbf{q})= & \sum_{\mathbf{k}}\left(v_{\mathbf{k}}^{2} u_{\mathbf{k}+\mathbf{q}}^{2} g_{\mathbf{k}, \mathbf{q}} g_{\mathbf{k}+\mathbf{q},-\mathbf{q}}-u_{\mathbf{k}} v_{\mathbf{k}} u_{-\mathbf{k}-\mathbf{q}} v_{-\mathbf{k}-\mathbf{q}} g_{\mathbf{k}, \mathbf{q}}^{2}\right) \times \\
& \times\left(\frac{1}{\omega-E_{\mathbf{k}}-E_{\mathbf{k}+\mathbf{q}}}+\frac{1}{-\omega-E_{\mathbf{k}}-E_{\mathbf{k}+\mathbf{q}}}\right) .
\end{aligned}
$$

In these formulas $g_{\mathbf{k}, \mathbf{q}}$ is hole-spin-wave vertex (6), and $u_{\mathbf{k}}, v_{\mathbf{k}}, E_{\mathbf{k}}$ are parameters of BCS hole wave function (11).

Let us consider now two and more loop corrections to polarization operator. Some diagrams of this type with normal hole Green's function are presented in Fig.5. One can easily prove that the diagram Fig.5a (single spin-wave exchange) vanishes due to the spin-flip nature of the hole-spin-wave vertex. Vanishing of this diagram is absolutely similar to the vanishing of the single loop correction to the hole-spin-wave vertex (see Refs. 31, 36, 25.). The diagram Fig.5b equals to zero for the same reason. To calculate the diagram Fig.5c let us represent it using Goldstone diagram technique: Fig.6. In the intermediate state in the diagram Fig.6a as well as in the diagram Fig.6b there is two-particles two-holes excitation 44]. Nevertheless these two diagrams are essentially different. All intermediate momenta in the diagram Fig.6b are in the vicinity of Fermi surface $\left(\mathbf{p}=\mathbf{k}-\mathbf{k}_{\mathbf{0}} \sim p_{F}\right)$, and therefore this diagram is convergent. On other hand the particles momenta in diagram Fig.6a are not restricted and this diagram is ultraviolet divergent. The divergence gives logarithmic enhancement of this diagram. Further we neglect contribution Fig.6b, and transform Fig.6a into an effective diagram Fig.7 with "dot" given by Fig.8.

We are interested in relatively small $q(q<1)$. Therefore all momenta in the diagram Fig.7 are close to the Fermi surface. It means that we need to calculate the effective interaction ("dot") at Fig.8 at $\mathbf{k} \approx \mathbf{k}+\mathbf{q} \approx( \pm \pi / 2, \pm \pi / 2)$ and $\mathbf{k}^{\prime} \approx \mathbf{k}^{\prime}+\mathbf{q} \approx( \pm \pi / 2, \pm \pi / 2)$. Let us neglect q-dependence (set $q=0$ ) and denote this effective interaction by $V^{(1)}\left(\mathbf{k}, \mathbf{k}^{\prime}\right)$. Single particle state near $\mathbf{k}_{0--}=(-\pi / 2,-\pi / 2)$ is related to that near $\mathbf{k}_{0++}=(\pi / 2, \pi / 2)$ via umklapp process and therefore these points are actually equivalent. It is like one pocket of the Fermi surface. Due to this reason $V^{(1)}\left(\mathbf{k}_{0++}, \mathbf{k}_{0++}\right)=V^{(1)}\left(\mathbf{k}_{0++}, \mathbf{k}_{0--}\right)$. Certainly one can prove this relation explicitly using perturbation theory expression corresponding to diagram Fig.8. So there are only two independent pockets of the Fermi surface (for example centred near $\mathbf{k}_{0++}$ and $\mathbf{k}_{0+-}$ ), and the effective interaction can be characterized by the two values only: 


$$
\begin{aligned}
& V_{++}^{(1)}=V^{(1)}\left(\mathbf{k}_{0++}, \mathbf{k}_{0++}\right), \\
& V_{+-}^{(1)}=V^{(1)}\left(\mathbf{k}_{0++}, \mathbf{k}_{0+-}\right) .
\end{aligned}
$$

First one is the effective particle-hole interaction when particle and hole are from the same pocket. Second value is particle-hole interaction when particle and hole are from different pockets.

Using hole-spin-wave interaction (6) one can easily derive expression for $V_{++}^{(1)}$ corresponding to diagram Fig.8

$$
V_{++}^{(1)}=-32 f^{4} \sum_{\mathbf{Q}} \frac{\gamma_{\mathbf{Q}+\mathbf{k}}^{2} \gamma_{\mathbf{Q}-\mathbf{k}}^{2}\left(4-\omega_{\mathbf{Q}}^{2}\right)}{\omega_{\mathbf{Q}}^{2}\left(\omega_{\mathbf{Q}}+E_{\mathbf{Q}+\mathbf{k}}\right)^{2} E_{\mathbf{Q}+\mathbf{k}}} .
$$

Here $\mathbf{k}=\mathbf{k}_{0++}$. At $p_{F}<Q<\pi$ the integral in (31) is logarithmic divergent $\left(\int d Q / Q\right)$ and therefore $V_{++}^{(1)}$ can be analytically calculated with logarithmic accuracy

$$
V_{++}^{(1)} \approx-\frac{2 f^{4}}{\pi \beta_{1}} \frac{\left(1+2 \sqrt{\left.\beta_{2} / \beta_{1}\right)}\right.}{\left(1+\sqrt{\beta_{2} / \beta_{1}}\right)^{2}} \cdot L .
$$

The big logarithm $L$ equals to

$$
L=\ln \left(\epsilon_{\Lambda} / \Delta\right),
$$

where $\Delta$ is typical value of superconducting gap and $\epsilon_{\Lambda} \sim 2$ is ultraviolet cutoff which is of the order of hole band width. Actually only this logarithm justifies introduction of the effective point-like interaction $V^{(1)}$. Certainly expression (31) can be easily exactly evaluated numerically. Similar consideration shows that due to the structure of hole-spin-wave vertex (6) effective particle hole interaction for particle and hole from different pockets vanishes

$$
V_{+-}^{(1)}=0
$$

Above discussion is not the end of the story about effective particle-hole interaction in the spin-flip channel. It was just a leading order. To find correct value we have to sum all the "ladder" given at Fig.9. Fortunately each additional rung in ladder gives additional big logarithm and therefore summation can be easily done with logarithmic accuracy

$$
V_{++}=V_{++}^{(1)}\left(1+\frac{f^{2}}{\pi \beta_{1}\left(1+\sqrt{\beta_{2} / \beta_{1}}\right)} \cdot L\right)^{-1} .
$$

Summation of the ladder does not change the relation (34): effective particle hole interaction for particle and hole from different pockets vanishes.

For very small hole concentration $\delta$ the superconducting gap $\Delta$ is approaching zero, $L \rightarrow \infty$, and hence

$$
V_{++} \rightarrow-2 f^{2} \frac{1+2 \sqrt{\beta_{2} / \beta_{1}}}{1+\sqrt{\beta_{2} / \beta_{1}}}
$$

In practice $L$ is never big enough to reach this limit. Realistic value of $L$ can be found by comparing (32) with the result of exact numerical calculation of (31). At $\delta=0.05 L \approx 4$, and it drops down to $L \approx 3$ at at $\delta=0.2$. Substituting correct value of $L$ into (35) we find renormalized particle-hole interaction in spin-flip channel $V_{++}$. Numerical values of $V_{++}$ 
are presented in the Table.II. Actually it is almost constant $V_{++} \approx-4.5$ for interesting concentrations.

Now we can calculate exact spin-wave polarization operator chaining effective particle hole interaction in the spin-flip channel, see Fig.10. This chaining is substantially simplified by the fact that the pockets of the Fermi surface are well separated. Let us represent single loop polarization operator (29) as a sum of contributions corresponding to different pockets

$$
\begin{aligned}
& \Pi^{(1)}=\Pi_{I}^{(1)}+\Pi_{I I}^{(1)} \\
& \Pi_{I}^{(1)}(\omega, \mathbf{q})=\sum_{\mathbf{k} \in I} X(\omega, \mathbf{k}, \mathbf{q}) \\
& \Pi_{I I}^{(1)}(\omega, \mathbf{q})=\sum_{\mathbf{k} \in I I} X(\omega, \mathbf{k}, \mathbf{q})
\end{aligned}
$$

where $X(\omega, \mathbf{k}, \mathbf{q})$ is the integrand in formula (29). Regions $I$ and $I I$ of magnetic Brillouin zone are shown at Fig.1. We remind that point $\mathbf{k}_{0--}=(-\pi / 2,-\pi / 2)$ is connected with $\mathbf{k}_{0++}=(\pi / 2, \pi / 2)$ via umklapp process and therefore the region $I$ represents one pocket. The same is valid for $I I$. Due to the condition (34) particle-hole scattering does not transfer particle-hole excitation from one pocket to another, but inside pocket scattering amplitude (35) is constant. In this situation summation of chain in Fig.10 is trivial. The exact spinwave polarization operator (28) is of the form

$$
\begin{aligned}
& \Pi(\omega, \mathbf{q})=\frac{\Pi_{I}^{(1)}(\omega, \mathbf{q})}{1+V_{++} Q_{I}(\omega, \mathbf{q})}+\frac{\Pi_{I I}^{(1)}(\omega, \mathbf{q})}{1+V_{++} Q_{I I}(\omega, \mathbf{q})} \\
& \bar{\Pi}(\omega, \mathbf{q})=\frac{\bar{\Pi}_{I}^{(1)}(\omega, \mathbf{q})}{1+V_{++} Q_{I}(\omega, \mathbf{q})}+\frac{\bar{\Pi}_{I I}^{(1)}(\omega, \mathbf{q})}{1+V_{++} Q_{I I}(\omega, \mathbf{q})},
\end{aligned}
$$

where

$$
Q_{I, I I}(\omega, \mathbf{q})=\sum_{\mathbf{k} \in I, I I}\left(v_{\mathbf{k}}^{2} u_{\mathbf{k}+\mathbf{q}}^{2}+u_{\mathbf{k}} v_{\mathbf{k}} u_{-\mathbf{k}-\mathbf{q}} v_{-\mathbf{k}-\mathbf{q}}\right)\left(\frac{1}{\omega-E_{\mathbf{k}}-E_{\mathbf{k}+\mathbf{q}}}+\frac{1}{-\omega-E_{\mathbf{k}}-E_{\mathbf{k}+\mathbf{q}}}\right) .
$$

Unfortunately further analytical calculation of $\Pi(\omega, \mathbf{q})$ and $\bar{\Pi}(\omega, \mathbf{q})$ given by (38) is impossible. However numerical calculation using (29), (37), (39) and parameters of superconducting wave function found in the Section II is straightforward. We will discuss it in detail in the next section. Let us look here only at the limit $q \rightarrow 0$. In this limit

$$
\Pi(\omega, \mathbf{q})=\Pi(-\omega, \mathbf{q})=-\bar{\Pi}(\omega, \mathbf{q}) .
$$

This reflects the fact that we can forget about all complications connected with multi component spin-wave Green's function (23), and use simple $\sigma$-model picture (13). Let us introduce parameter of spin-wave instability

$$
C=\frac{-2 \Pi(0, \mathbf{q})}{\omega_{\mathbf{q}}}, \mathbf{q} \rightarrow 0
$$

According to equation (14) if $C>1$ spectrum of excitations is unstable. Naive value of $C$ corresponding to normal Fermi liquid approximation (26) is

$$
C_{\text {naive }}=\frac{2 f^{2}}{\pi \sqrt{\beta_{1} \beta_{2}}} \approx 2.8 \text {. }
$$

Numerical values of $C$ obtained with (38) for different hole concentrations are presented in the Table II. We see that pairing and particle-hole rescattering (35) substantially reduce value of $C$. Actually rescattering is more important, but it has the same origin as the pairing. Nevertheless $C>1$, and long-range antiferromagnetic order is unstable. 


\section{SPIN-WAVE GREEN'S FUNCTION.}

To deal with spin-wave instability let us apply approach developed in the Section III. With Takahashi condition (20),(18) bare spin-wave Hamiltonian is transformed to

$$
\begin{aligned}
& \sum_{\mathbf{q}} \omega_{\mathbf{q}}\left(\alpha_{\mathbf{q}}^{\dagger} \alpha_{\mathbf{q}}+\beta_{\mathbf{q}}^{\dagger} \beta_{\mathbf{q}}\right) \rightarrow \sum_{\mathbf{q}} \omega_{\mathbf{q}}\left(\alpha_{\mathbf{q}}^{\dagger} \alpha_{\mathbf{q}}+\beta_{\mathbf{q}}^{\dagger} \beta_{\mathbf{q}}\right)-\frac{1}{8} \nu^{2}\left(S_{u}^{z}-S_{d}^{z}\right) \rightarrow \\
& \rightarrow \sum_{\mathbf{q}}\left[\left(\omega_{\mathbf{q}}+\frac{\nu^{2}}{2 \omega_{\mathbf{q}}}\right)\left(\alpha_{\mathbf{q}}^{\dagger} \alpha_{\mathbf{q}}+\beta_{\mathbf{q}}^{\dagger} \beta_{\mathbf{q}}\right)-\gamma_{\mathbf{q}} \frac{\nu^{2}}{2 \omega_{\mathbf{q}}}\left(\alpha_{\mathbf{q}} \beta_{-\mathbf{q}}+\alpha_{\mathbf{q}}^{\dagger} \beta_{-\mathbf{q}}^{\dagger}\right)\right] .
\end{aligned}
$$

Lagrange multiplier $\nu$ will be found later from the condition (20). Dioganalization of the Hamiltonian (43) by usual Bogoliubov transformation gives new bare spin-wave spectrum

$$
\omega_{\nu \mathbf{q}}^{2}=\omega_{\mathbf{q}}^{2}+\nu^{2}+\frac{\nu^{4}}{16} \approx \omega_{\mathbf{q}}^{2}+\nu^{2}
$$

and new bare spin-wave operators $A_{\mathbf{q}}^{\dagger}, B_{\mathbf{q}}^{\dagger}$

$$
\begin{aligned}
& \alpha_{\mathbf{q}}=c_{\mathbf{q}} A_{\mathbf{q}}+s_{\mathbf{q}} B_{-\mathbf{q}}^{\dagger}, \\
& \beta_{-\mathbf{q}}=s_{\mathbf{q}} A_{\mathbf{q}}^{\dagger}+c_{\mathbf{q}} B_{-\mathbf{q}}, \\
& c_{\mathbf{q}}, s_{\mathbf{q}}=\frac{\omega_{\nu \mathbf{q}} \pm \omega_{\mathbf{q}}}{2 \omega_{\nu \mathbf{q}} \omega_{\mathbf{q}}}
\end{aligned}
$$

We will see below that $\nu$ is very small. Therefore we neglect $\nu^{4} / 16$ in comparison with $\nu^{2}$. Hole-spin-wave interaction (6) should be also expressed in terms of $A_{\mathbf{q}}$ and $B_{\mathbf{q}}$ operators

$$
\begin{aligned}
& H_{h, s w}=\sum_{\mathbf{k}, \mathbf{q}} G_{\mathbf{k}, \mathbf{q}}\left(h_{\mathbf{k}+\mathbf{q} \downarrow}^{\dagger} h_{\mathbf{k} \uparrow} A_{\mathbf{q}}+h_{\mathbf{k}+\mathbf{q} \uparrow}^{\dagger} h_{\mathbf{k} \downarrow} B_{\mathbf{q}}+\text { H.c. }\right), \\
& G_{\mathbf{k}, \mathbf{q}}=c_{\mathbf{q}} g_{\mathbf{k}, \mathbf{q}}+s_{\mathbf{q}} g_{\mathbf{k}+\mathbf{q},-\mathbf{q}} .
\end{aligned}
$$

Let us note that due to the Goldstone theorem $g_{\mathbf{k}, \mathbf{q}} \rightarrow 0$ at $\mathbf{q} \rightarrow 0$. However, in the state which we discuss now rotational symmetry is restored and therefore $G_{\mathbf{k}, \mathbf{0}}=\sqrt{2} f \gamma_{\mathbf{k}}$.

Similar to (23) we introduce Green's functions $D_{A A}(t, \mathbf{q}), D_{A B}(t, \mathbf{q}), D_{B A}(t, \mathbf{q})$ and $D_{B B}(t, \mathbf{q})$. Zero approximation Green's functions $D_{A A}^{0}(\omega, \mathbf{q}) \ldots$ are given by eqs. (24) with replacement $\omega_{\mathbf{q}} \rightarrow \omega_{\nu \mathbf{q}}$. Polarization operators are given by the formulas of the previous section with replacement $g_{\mathbf{k}, \mathbf{q}} \rightarrow G_{\mathbf{k}, \mathbf{q}}$. Spin-wave Green's functions obey usual Dyson equations 40,41)

$$
\begin{aligned}
& D_{A A}=D_{A A}^{(0)}+D_{A A}^{(0)} P_{A A} D_{A A}+D_{A A}^{(0)} P_{A B} D_{B A}, \\
& D_{B B}=D_{B B}^{(0)}+D_{B B}^{(0)} P_{B B} D_{B B}+D_{B B}^{(0)} P_{B A} D_{A B}, \\
& D_{A B}=D_{A A}^{(0)} P_{A A} D_{A B}+D_{A A}^{(0)} P_{A B} D_{B B}, \\
& D_{B A}=D_{B B}^{(0)} P_{B A} D_{A A}+D_{B B}^{(0)} P_{B B} D_{B A} .
\end{aligned}
$$

Solution of these equations together with (28) gives

$$
\begin{aligned}
& D_{A A}(\omega, \mathbf{q})=D_{B B}(-\omega, \mathbf{q})=\frac{-\omega-\omega_{\mathbf{q}}-\Pi(-\omega)}{\lambda(\omega, \mathbf{q})} \\
& D_{A B}(\omega, \mathbf{q})=D_{B A}(\omega, \mathbf{q})=\frac{\bar{\Pi}(\omega)}{\lambda(\omega, \mathbf{q})} \\
& \lambda(\omega, \mathbf{q})= \\
& =-\omega^{2}+\omega_{\nu \mathbf{q}}^{2}+\omega_{\nu \mathbf{q}}(\Pi(\omega)+\Pi(-\omega))+\omega(\Pi(\omega)-\Pi(-\omega))+\Pi(\omega) \cdot \Pi(-\omega)-\bar{\Pi}^{2}(\omega) .
\end{aligned}
$$


In these formulas we omit for simplicity argument $\mathbf{q}$ in the polarization operator. We do not specify imaginary part of $\lambda$ in the poles because below we perform Wick's rotation $\omega \rightarrow i \xi$ which automatically gives correct behaviour. Using (18) one can easily express average magnetization in the terms of Green's functions (49)

$$
\begin{aligned}
0 & =\left\langle\frac{1}{2}\left(S_{u}^{z}-S_{d}^{z}\right)\right\rangle=1-2 i \sum_{\mathbf{q}} \frac{1}{\omega_{\nu \mathbf{q}}}\left\{D_{A A}(t=-0, \mathbf{q})+D_{B B}(t=-0, \mathbf{q})-\right. \\
& \left.-\gamma_{\mathbf{q}}\left(D_{A B}(t=-0, \mathbf{q})+D_{B A}(t=-0, \mathbf{q})\right)\right\}= \\
& =1-2 \sum_{\mathbf{q}} \frac{1}{\Omega_{\nu \mathbf{q}}}=0.303-2 \sum_{\mathbf{q}}\left(\frac{1}{\Omega_{\nu \mathbf{q}}}-\frac{1}{\omega_{\mathbf{q}}}\right)
\end{aligned}
$$

where $\Omega_{\nu \mathbf{q}}$ is given by the expression

$$
\frac{1}{\Omega_{\nu \mathbf{q}}}=\frac{1}{\omega_{\nu \mathbf{q}}} \int_{-\infty}^{+\infty} \frac{d \xi}{2 \pi} \frac{1}{\lambda(i \xi, \mathbf{q})}\left(2 \omega_{\nu \mathbf{q}}+\left[\Pi(i \xi, \mathbf{q})+\Pi(-i \xi, \mathbf{q})+2 \gamma_{\mathbf{q}} \bar{\Pi}(i \xi, \mathbf{q})\right]\right) .
$$

We have to find the Lagrange multiplier $\nu$ from equation (51). At small $\mathbf{q}$, due to the relation (40), the expression in square brackets in (52) vanishes and $\lambda(i \xi, \mathbf{q}) \rightarrow \xi^{2}+\omega_{\nu \mathbf{q}}^{2}+2 \omega_{\nu \mathbf{q}} \Pi(i \xi, \mathbf{q})$. If one neglects also $\xi$-dependence of polarization operator, the integration over $\xi$ in (52) is trivial and it is reduced to formula (22) derived in Section III in $\sigma$-model approximation.

Now we can proceed to the discussion of the results of numerical calculations. Dependence of hole-hole pairing on the modification of spin-wave spectrum is rather weak at $\delta \ll 1$, and we neglect it (see also end of these section). Therefore, we use parameters of hole BCS wave function found in Section II. Calculation of the polarization operator is described in detail in Section IV. One should not forget about the replacement $g_{\mathbf{k}, \mathbf{q}} \rightarrow G_{\mathbf{k}, \mathbf{q}}$, but numerically it is not very important. The values of $\nu$ found from equation (51) are presented in the Table II. The effective spin-wave frequency $\Omega_{\nu \mathbf{q}}$ depends both on direction and magnitude of $\mathbf{q}$. It is convenient to take the bare spin-wave frequency $\omega_{\mathbf{q}}$ as an argument instead of $|\mathbf{q}|$. The plots of $\Omega_{\nu \mathbf{q}}$ as a function of $\omega_{\mathbf{q}}$ for the directions $\mathbf{q} \propto(1,0)$ and $\mathbf{q} \propto(1,1)$ and for different hole concentrations are given in Figs.11a-d.

At very small hole concentration $\delta$, equation (51) has no solution. One can easily understand the reason for this. The parameter of spin-wave instability $C$ is larger then 1 at arbitrary small hole concentration $\delta$, see eqs.(41),(42) and Table II. It means that dependence of $\Omega_{\nu \mathbf{q}}$ on $|\mathbf{q}|$ should be of the type of solid line at Fig.11a with minimum at $q_{0} \sim p_{F}$. Let us denote the value of $\Omega_{\nu \mathbf{q}}$ at this point by $\Omega_{0}$. This minimum value is directly related to $\nu$. Therefore instead of $\nu$ we can consider $\Omega_{0}$ as a variable which should be found from eq.(51). If frequency $\Omega_{\nu \mathbf{q}}$ is isotropic $\Omega_{\nu \mathbf{q}}^{2} \approx \Omega_{0}^{2}+a\left(q-q_{0}\right)^{2}$ the equation (51) has obvious solution at arbitrary small $\delta$

$$
\Omega_{0} \sim e^{-1 / q_{0}} \sim e^{-1 / p_{F}} \sim e^{-1 / \sqrt{\delta}}
$$

The problem is that the polarization operator and hence $\Omega_{\nu \mathbf{q}}^{2}$ is not isotropic. It is seen explicitly from Fig. 11a. The anisotropy is proportional to some power of $q_{0} \propto \sqrt{\delta}$. Therefore at small enough concentration the anisotropy is larger than exponentially small $\Omega_{0}$ and equation (51) has no solution. Thus if we start from spin-liquid phase (say from $\delta=0.1$ ) and decrease hole density $\delta$, at some critical value $\delta=\delta_{c}$ the gap $\Omega_{0}$ vanishes. It means instability of spin liquid state with respect to decay to spin waves with momentum $\mathbf{q}_{\mathbf{0}}$ (spirals). The critical value $\delta_{c} \approx 3-4 \%$. Due to the strong exponential dependence of (53) on $\delta$ it is hard to find numerically the exact value of $\delta_{c}$. We would like to note that the dip in Fig.11a is a result of virtual admixture of spirals to the spin-liquid ground state. 
One can consider $\nu$ as a gap $\Delta_{M}$ in the spin-wave spectrum at $q=0$. At small hole concentration $(\delta=0.05,0.1)$ minimum of the effective spectrum is shifted from the point $q=0$, see Figs.11ab. One has to be careful with interpretation of $\Delta_{M}$. The effective frequency $\Omega_{\nu \mathbf{q}}^{2}$ defined by eq.(52) is not a frequency of simple excitation in the sense that Green's function $G \propto 1 /\left(\omega^{2}-\Omega_{\nu \mathbf{q}}^{2}\right)$. Green's function is of essentially more complicated form. It has poles and cuts. Therefore, using standard terminology, it is better to say that $\Delta_{M}$ is pseudo-gap and that $\Omega_{\nu \mathbf{q}}$ is pseudo-spectrum. The calculated value of pseudo-gap as well as its dependence on the hole concentration reasonably agrees with experimental data 45 48.

In equation (51) we have overcome the problem of real spectrum by integrating over imaginary $\omega$. For real $\omega$ structure of the spectrum is as follow. At very small $q \ll p_{F}$ there is a damped spin-wave with frequency $\omega \approx \Delta_{M} \approx \nu$. The decay of the spin-wave into particle-hole pair is allowed due to the nodes of the superconducting gap. With increasing of $q$, spectrum is split into the two branches: 1)damped spin-wave with frequency $\omega \sim \omega_{\mathbf{q}}$, and 2)low frequency collective excitation consisting mainly of the particles and holes. Starting from some value of $q$ decay of this excitation into particle and hole is forbidden due to the superconducting gap. Therefore in this low frequency region spin-wave Green's function can be represented as

$$
G(\omega, \mathbf{q})=\frac{Z}{\omega^{2}-o_{\mathbf{q}}^{2}}
$$

where $Z$ is the spin-wave residue. The frequency of collective excitation $o_{\mathbf{q}}$ as a function of $\omega_{\mathbf{q}}$ for direction $\mathbf{q} \propto(1,0)$ and for hole concentration $\delta=0.1$ is plotted at Fig.11b. Due to the decay to real particle-hole pair this spectrum has no meaning at very small $q$ and at large $q$. Spin-wave residue $Z$ changes along the drawn curve from $Z=0.75$ at left hand side of curve to $Z=0.15$ at right hand side of the interval. Detail analysis of the spectrum of spin $S=1$ excitations is an important problem. It requires calculation of the dynamic magnetic formfactor $S_{M}(\omega, \mathbf{q})$ of the system. Having this formfactor one can perform accurate comparison with an experimental data on neutron scattering and NMR. It is a subject of a separate work. Here we concentrate on the magnetic correlation length $\xi_{M}$ and static magnetic formfactor $S_{M}(\mathbf{q})$.

This is the place to remind the reader that in the calculation of the superconducting pairing we have used unperturbed spin-wave spectrum. This approximation is justified by the parameter $\sqrt{\delta} \ll 1$ (see Refs. [1].2] and eq.(9)). However for most interesting concentrations $(\delta \sim 0.15-0.2)$ this parameter is not so good. We see from Fig.11a that for $\delta=0.05$ the effective spin-wave spectrum is actually close to the unperturbed one. However for $\delta=0.2$ (Fig.11d) deviation is rather big. Therefore to complete our program we have to solve Eliashberg equations for pairing with renormalized spin-wave Green's function (compare with Ref. 49]). This should be a subject of a separate work. However qualitatively the influence of this effect is evident. Effective pairing interaction (7) is roughly proportional to the inverse spin-wave frequency. Therefore, the renormalized interaction gives stronger pairing than that in "zero approximation". Due to our preliminary estimations, the correction to pairing is about $30 \%$ at $\delta=0.2$.

\section{MAGNETIC CORRELATION LENGTH AND STATIC MAGNETIC FORMFACTOR}

We remind the reader that the spin wave operators $\alpha_{\mathbf{q}}$ and $\beta_{\mathbf{q}}$ are introduced using Dyson-Maleev transformation [50 for localized spin $\mathbf{S}$, 


$$
\begin{aligned}
& S_{l}^{-}=a_{l}^{\dagger}, \quad S_{l}^{+}=\left(2 S-a_{l}^{\dagger} a_{l}\right) a_{l}, \\
& S_{l}^{z}=S-a_{l}^{\dagger} a_{l}, \quad \text { for } \quad l \in \text { up sublattice} ; \\
& S_{m}^{-}=b_{m}, \quad S_{m}^{+}=b_{m}^{\dagger}\left(2 S-b_{m}^{\dagger} b_{m}\right), \\
& S_{m}^{z}=-S+b_{m}^{\dagger} b_{m}, \quad \text { for } m \in \text { dawn } \quad \text { sublattice. }
\end{aligned}
$$

The operators $\alpha_{\mathbf{q}}$ and $\beta_{\mathbf{q}}$ are related to $a_{l}$ and $b_{m}$ by usual combination of Fourier and Bogoliubov transformations, see e.g. Refs. [38,28]. Following Takahashi [38] we introduce notations

$$
\begin{aligned}
f\left(\mathbf{r}_{l}-\mathbf{r}_{l^{\prime}}\right) & =\left\langle a_{l}^{\dagger} a_{l^{\prime}}\right\rangle, \quad l \neq l^{\prime}, \\
f\left(\mathbf{r}_{m}-\mathbf{r}_{m^{\prime}}\right) & =\left\langle b_{m}^{\dagger} b_{m^{\prime}}\right\rangle, \quad m \neq m^{\prime}, \\
g\left(\mathbf{r}_{l}-\mathbf{r}_{m}\right) & =\left\langle a_{l}^{\dagger} b_{m}^{\dagger}\right\rangle=\left\langle a_{l} b_{m}\right\rangle,
\end{aligned}
$$

where brackets $\langle\ldots\rangle$ represents an average over ground state. Using mean field procedure for the averaging of the quartic terms $\left(\left\langle a^{\dagger} a b^{\dagger} b\right\rangle=\left\langle a^{\dagger} a\right\rangle\left\langle b^{\dagger} b\right\rangle+\left\langle a^{\dagger} b^{\dagger}\right\rangle\langle a b\rangle\right)$ one can express spin-spin correlators in terms of functions $f$ and $g$ (see Ref. [38]).

$$
\begin{aligned}
& \left\langle\mathbf{S}_{i} \mathbf{S}_{j}\right\rangle=f^{2}\left(\mathbf{r}_{i}-\mathbf{r}_{j}\right)-\frac{1}{4} \delta_{i, j}, \quad i, j \in \text { same sublattice, } \\
& \left\langle\mathbf{S}_{i} \mathbf{S}_{j}\right\rangle=-g^{2}\left(\mathbf{r}_{i}-\mathbf{r}_{j}\right), \quad i, j \in \text { different sublattices. }
\end{aligned}
$$

Using definition (56), the functions $f$ and $g$ can be expressed in terms of Green's functions $D_{A A}(t=-0, \mathbf{q}), D_{A B}(t=-0, \mathbf{q}) \ldots$ This is quite similar to the average magnetization (51). Then with the help of formulas (49) one gets

$$
\begin{aligned}
& f(\mathbf{r})=2 \sum_{\mathbf{q}} \frac{e^{i \mathbf{q} \mathbf{r}}}{\Omega_{\nu \mathbf{q}}}, \\
& g(\mathbf{r})=-2 \sum_{\mathbf{q}} \frac{e^{i \mathbf{q r}}}{\Omega_{\nu \mathbf{q}}^{\prime}},
\end{aligned}
$$

The effective frequency $\Omega_{\nu \mathbf{q}}$ is given by equation (52). The "prime" effective frequency is defined by slightly different formula

$$
\frac{1}{\Omega_{\nu \mathbf{q}}^{\prime}}=\frac{1}{\omega_{\nu \mathbf{q}}} \int_{-\infty}^{+\infty} \frac{d \xi}{2 \pi} \frac{1}{\lambda(i \xi, \mathbf{q})}\left(\gamma_{\mathbf{q}}\left[2 \omega_{\nu \mathbf{q}}+\Pi(i \xi, \mathbf{q})+\Pi(-i \xi, \mathbf{q})\right]+2 \bar{\Pi}(i \xi, \mathbf{q})\right) .
$$

For small momentum $\gamma_{\mathbf{q}} \approx 1$. Therefore $\Omega_{\nu \mathbf{q}}^{\prime} \approx \Omega_{\nu \mathbf{q}}$ for $q \ll 1$. According to computations the relation $1 / \Omega_{\nu \mathbf{q}}^{\prime} \approx \gamma_{\mathbf{q}} / \Omega_{\nu \mathbf{q}}$ is valid with reasonable accuracy for arbitrary $q$. Let us note that due to the spin-liquid equation (51) $f(0)=1$. Together with (57) this gives correct value of spin: $\left\langle\mathbf{S}^{2}\right\rangle=3 / 4$.

The results of numerical calculation of the functions $f$ and $g$ are given in Fig.12. Distance between sites attains the values $r=\sqrt{m^{2}+n^{2}}$. We plot $\ln g(r)$ for odd value of $m+n$, and $\ln f(r)$ for even value of $m+n$. One can see that for $r>2$ the dependence is practically linear. Hence

$$
\langle\mathbf{S}(t, \mathbf{r}) \cdot \mathbf{S}(t, 0)\rangle \propto(-1)^{m+n} \exp \left(-r / \xi_{M}\right), \quad \text { for } r>2 .
$$

The values of the magnetic correlation length $\xi_{M}$ found from Fig.12 are given in the Table II.

Using eqs.(57) and (58) one can express static magnetic formfactor in terms of the effective frequencies $\Omega_{\nu \mathbf{k}}$ and $\Omega_{\nu \mathbf{k}}^{\prime}$ 


$$
S_{M}(\mathbf{q})=\sum_{\mathbf{r}} \exp (i \mathbf{q} \cdot \mathbf{r})\left\langle\mathbf{S}(t, \mathbf{r}) \cdot \mathbf{S}(t, 0)=-\frac{1}{4}+2 \sum_{\mathbf{k}}\left(\frac{1}{\Omega_{\nu \mathbf{k}} \Omega_{\nu \mathbf{k}+\mathbf{q}}}-\frac{1}{\Omega_{\nu \mathbf{k}}^{\prime} \Omega_{\nu \mathbf{k}+\mathbf{q}}^{\prime}}\right)\right.
$$

For $\mathbf{q}=0$ the integrand in (61) vanishes at small $\mathbf{k}$ because $\Omega_{\nu \mathbf{k}}=\Omega_{\nu \mathbf{k}}^{\prime}$ at $k \ll 1$. Therefore only large momenta contribute into $S_{M}(0)$. For $\mathbf{q}=\mathbf{Q}=(\pi, \pi)$ situation is different because $\Omega_{\nu \mathbf{k}+\mathbf{Q}}=\Omega_{\nu \mathbf{k}}$ and $\Omega_{\nu \mathbf{k}+\mathbf{Q}}^{\prime}=-\Omega_{\nu \mathbf{k}}^{\prime}$. Therefore contribution of small $k$ into $S_{M}(\mathbf{Q})$ is stressed like one over spin-wave pseudo-gap squared $\left(1 / \nu^{2}\right)$. The values of $S_{M}(0)$ and $S_{M}(\mathbf{Q})$ for different hole concentrations are given in the Table II. The static formfactor $S_{M}(\mathbf{q})$ at hole concentration $\delta=0.15$ is plotted at Fig.13. We have to note that due to the strong dependence on the pseudo-gap which is nearly zero at $\delta=0.05$, the value of $S_{M}(\mathbf{Q})$ at this concentration is sensitive to small variation of parameters. Therefore the accuracy of calculation of $S_{M}(\mathbf{Q})$ at this point is very poor. This is reflection of the closeness to the critical concentration $\delta_{c}$ at which system drops down into the irregular spin-glass state (see discussion in the previous Section).

Although we do not observe the shift of the $S_{M}(\mathbf{q})$ maximum from the point $\mathbf{Q}=(\pi, \pi)$ our result agrees qualitatively with that of finite cluster numerical simulation [51] (see also review paper [29] and references therein, where results for other models are presented). However the values of $S_{M}(\mathbf{Q})$ obtained by us are substantially higher than those from finite cluster simulations. We have mentioned already that $S_{M}(\mathbf{Q})$ is very sensitive to the spinwave pseudo-gap at small momenta. Therefore the possible reason of disagreement is that with relatively small claster it is impossible to reproduce correct spin-wave spectrum at small momenta.

\section{CONCLUSION}

In the present work using the picture of spin-wave condensation we have derived explicitly spin liquid ground state of the doped two-dimensional $t-J$ model. Translation invariant spin-liquid solution exists at doping $\delta \geq \delta_{c} \approx 3-4 \%$. We have demonstrated that spin-wave condensation and condensation of Cooper pairs (superconducting pairing of mobile holes) are intrinsically connected. The developed approach allows to calculate any property of the ground state as well as spectrum of excitations. In the present work we have calculated spin-wave pseudo-gap $\Delta_{M}$ (Table II), effective spectrum (or pseudo-spectrum) of $S=1$ excitations (Fig.11), magnetic correlation length $\xi_{M}$ and static magnetic formfactor $S_{M}(\mathbf{q})$ (Table II and Fig. 13).

\section{ACKNOWLEDGMENTS}

We have derived the basic results presented in the section III together with M. Yu. Kuchiev. I am also very grateful to him for numerous helpful discussions. I thank V. V. Flambaum, G. F. Gribakin and A. V. Dotsenko for critical and stimulating discussions. 


\section{REFERENCES}

a Also at the Budker Institute of Nuclear Physics, 630090 Novosibirsk, Russia

[1] V. V. Flambaum, M. Yu. Kuchiev, O. P. Sushkov. Physica C 227, 267 (1994).

[2] V. I. Belinicher, A. L. Chernyshev, A. V. Dotsenko, O. P. Sushkov. Phys. Rev. B, 51, 6076 (1995).

[3] J. R. Schrieffer, X. C. Wen, and S. C. Zhang, Phys. Rev. Lett 60, 944 (1988).

[4] N. E. Bickers, D. J. Scalapino, S. R. White, Phys. Rev. Lett. 62, 961 (1989).

[5] A. Kampf and J. R. Schrieffer, Phys. Rev. B 41, 6399 (1990).

[6] N. Bulut and D. J. Scalapino, Phys. Rev. B 45, 2371 (1992).

[7] P. Monthoux, A. V. Balatsky, and D. Pines, Phys. Rev. Lett. 67, 3448 (1991); Phys. Rev. B 46, 14803 (1992).

[8] P. Monthoux and D. Pines, Phys. Rev. Lett. 69, 961 (1992); Phys. Rev. B 47, 6097 (1993); Phys. Rev. B 49, 4277 (1994).

[9] E. Dagotto, A. Nazarenko, and A. Moreo, Phys. Rev. Lett. 74, 310 (1995).

[10] P. W. Anderson, Science 235, 1196 (1987).

[11] B. Shraiman and E. Siggia, Phys. Rev. Lett. 62,1564 (1989).

[12] T. Dombre, J. Physique 51, 847 (1990).

[13] A. Singh and Z. Tesanovic, Phys. Rev. B 41, 614 (1990).

[14] R. Eder, Phys. Rev. B 43, 10706 (1991).

[15] J. Igarashi and P. Fulde, Phys. Rev. B 45, 10419 (1992).

[16] O. P. Sushkov and V. V. Flambaum, Physica C 206, 269 (1993).

[17] A. V. Chubukov and S. Sachdev, Phys. Rev. Lett. 71, 169 (1993).

[18] A. Sokol and D. Pines, Phys. Rev. Lett. 71, 2813 (1993).

[19] S. Sachdev, Phys. Rev. B 49, 6770 (1994).

[20] A. V. Chubukov, S. Sachdev and J. Ye, Phys. Rev. B 49, 11919 (1994).

[21] J. E. Hirch, Phys. Rev. Lett. 54, 1317 (1985).

[22] C. Gross, R. Joynt, and T. M. Rice, Phys. Rev. B 36, 381 (1987).

[23] J. Oitmaa and D. D. Betts, Can. J. Phys. 56, 897 (1971).

[24] D. A. Huse, Phys. Rev. B 37, 2380 (1988).

[25] O. P. Sushkov, Phys. Rev. B 49, 1250 (1994).

[26] A. L. Chernyshev, A. V. Dotsenko, and O. P. Sushkov, Phys. Rev. B 49, 6197 (1994).

[27] M. Yu. Kuchiev and O. P. Sushkov, Physica C 218, 197 (1993).

[28] E. Manousakis, Rev. Mod. Phys. 63, 1 (1991).

[29] E. Dagotto, Rev. Mod. Phys. 66, 763 (1994) and references therein.

[30] O. P. Sushkov, Solid State Communications 83, 303 (1992).

[31] G. Martinez and P. Horsch, Phys. Rev. B 44, 317 (1991).

[32] T. Giamarchi and C. Lhuillier, Phys.Rev. B 47, 2775 (1993).

[33] H. Eskes, G. A. Sawatzky, and L. F. Feiner, Physica C 160, 424 (1989).

[34] V. V. Flambaum and O. P. Sushkov, Physica C 175, 347 (1991).

[35] V. I. Belinicher and A. L. Chernyshev, Phys. Rev. B 47, 390 (1993).

[36] Z. Liu and E. Manousakis, Phys. Rev. B 45, 2425 (1992).

[37] C. L. Kane, P. A. Lee, and N. Read, Phys. Rev. B 39, 6880 (1989).

[38] M. Takahashi Phys. Rev. B 40, 2494 (1989).

[39] A. V. Dotsenko and O. P. Sushkov, Phys. Rev. B 50, 13821 (1994).

[40] J. Igarashi and P. Fulde, Phys. Rev. B 45, 12357 (1992).

[41] G. Khaliullin and P. Horsch, Phys. Rev. B 47, 463 (1993).

[42] K. W. Becker and U. Muschelknautz, Phys. Rev. B 48, 13826 (1993).

[43] D. Murray and O. P. Sushkov, to be published. 
[44] Sometimes we use term "hole" in sense of $t-J$ model and sometimes (usually in combination particle-hole) in sense of Green's function technique. In each particular case the meaning of term is evident from context and can not cause misunderstanding.

[45] J. Tranquada et al, Phys. Rev. B 46, 5561 (1992).

[46] J. Rossat-Mignod et al, Physica B 192, 109 (1993).

[47] M. Sato et al, J. Phys. Soc. Jpn. 62, 263 (1993).

[48] L. P. Regnault et al. Physica C 235-240, 59 (1994).

[49] N. M. Plakida, P. Horsch, A. Liechtenstein, and V. S. Oudovenko, Preprint JINR, E17-95-287, Dubna, 1995.

[50] F. J. Dyson, Phys. Rev. 102, 1217,1230 (1956); S. V. Maleev, Zh. Eksp. Teor. Fiz. 30, 1010 (1957) [Sov. Phys. JETP 6, 776 (1958).

[51] A. Moreo, E. Dagotto, T. Jolicoeur, and J. Riera, Phys. Rev. B 42, 6283 (1990). 
TABLES

TABLE I. d-wave pairing at $t=3, \beta_{1} / \beta_{2}=7, f=1.8$ and different values of hole concentration $\delta$. Units correspond to $J=1$. The values of 1)Fermi energy $\epsilon_{F}, 2$ ) Chemical potential at zero temperature $\mu(0), 3)$ Maximum value of the gap in Brillouin zone at zero temperature $\Delta_{\max }(0)$, 4)Maximum value of the gap at Fermi surface at zero temperature $\left.\Delta_{\text {Fmax }}(0), 5\right)$ Chemical potential at critical temperature $\left.\mu\left(T_{c}\right), 6\right)$ Critical temperature $T_{c}$. For guidance we give in brackets the value in degrees, assuming that $J=0.15 \mathrm{eV}$.

\begin{tabular}{lcllllc}
\hline \hline$\delta$ & $\epsilon_{F}$ & $\mu(0)$ & $\Delta_{\max }(0)$ & $\Delta_{F \max }(0)$ & $\mu\left(T_{c}\right)$ & $T_{c}$ \\
\hline 0.05 & 0.054 & 0.049 & 0.033 & 0.031 & 0.053 & $0.016\left(28^{\circ}\right)$ \\
0.1 & 0.103 & 0.091 & 0.067 & 0.056 & 0.097 & $0.029\left(50^{\circ}\right)$ \\
0.15 & 0.144 & 0.125 & 0.102 & 0.079 & 0.130 & $0.040\left(70^{\circ}\right)$ \\
0.20 & 0.178 & 0.155 & 0.130 & 0.097 & 0.160 & $0.048\left(84^{\circ}\right)$ \\
\hline \hline
\end{tabular}

TABLE II. Parameters relevant to spin-wave spectrum at $t=3, \beta_{1} / \beta_{2}=7, f=1.8$ and different values of hole concentration $\delta$. Units correspond to $J=1$. 1) Renormalized particle-hole interaction in spin-flip channel $V_{++} .2$ ) The parameter of spin-wave instability $C$ defined by (41). 3) Lagrange parameter $\nu$ which is equal to the pseudo-gap $\Delta_{M}$ in the spin-wave spectrum. In the brackets we give the value in degrees, assuming that $J=0.15 \mathrm{eV}$. 4) Magnetic correlation length $\xi_{M}$ in the units of lattice spacing. 5) Static magnetic formfactor $S_{M}(\mathbf{q})$ for $\mathbf{q}=0$. 6) Static magnetic formfactor $S_{M}(\mathbf{q})$ for $\mathbf{q}=\mathbf{Q}=(\pi, \pi)$

\begin{tabular}{lcccccc}
\hline \hline$\delta$ & $V_{++}$ & $C$ & $\nu=\Delta_{M}$ & $\xi_{M}$ & $S(0)$ & $S_{M}(\mathbf{Q})$ \\
\hline 0.05 & -5.0 & 1.18 & $0.048\left(84^{\circ}\right)$ & 3.4 & 0.035 & $\sim 12$. \\
0.1 & -4.7 & 1.14 & $0.057\left(99^{\circ}\right)$ & 2.2 & 0.066 & 7.9 \\
0.15 & -4.5 & 1.10 & $0.075\left(130^{\circ}\right)$ & 1.6 & 0.088 & 5.0 \\
0.20 & -4.3 & 1.05 & $0.098\left(171^{\circ}\right)$ & 1.3 & 0.11 & 3.7 \\
\hline \hline
\end{tabular}




\section{FIGURE CAPTIONS}

FIG. 1. Magnetic Brillouin zone and the contour plot of a single hole mean occupation number $v_{\mathbf{k}}^{2}$ at $\delta=0.1$ and zero temperature.

FIG. 2. Single spin-wave exchange mechanism of hole-hole attraction.

Fig. 3.The spin-wave polarization operator in the Schrodinger representation:

a) One spin-wave is annihilated and the other one is created.

b,c)Two spin-waves are either annihilated or created.

FIG. 4. Polarization operator $P_{\alpha \alpha}(\omega, \mathbf{q})$ in one loop approximation. a - contribution of the normal hole Green's function, b - contribution of the anomalous hole Green's function.

FIG. 5. Corrections to the polarization operator. a)Single spin-wave exchange, b,c)double spin wave exchange.

FIG. 6. The diagram Fig.5c represented in the Goldstone diagram technique.

FIG. 7. Transform of the diagram Fig.6a into diagram with effective "dot" interaction.

FIG. 8. Effective "dot".

FIG. 9. The effective particle-hole interaction in the spin-flip channel.

FIG. 10. Spin-wave polarization operator with chaining of effective particle hole interaction taken into account.

FIG. 11. Effective spin-wave frequency $\Omega_{\nu \mathbf{q}}$ as a function of the bare frequency $\omega_{\mathbf{q}}$.

Solid line: the direction $\mathbf{q} \propto(1,0)$. Dashed line: the direction $\mathbf{q} \propto(1,1)$. For comparison we present unrenormalized frequency $\left(\Omega_{\nu \mathbf{q}}=\omega_{\mathbf{q}}\right)$ : dashed-dotted line.

a): hole concentration $\delta=0.05$.

b): $\delta=0.1$. For this concentration we present also the frequency of collective excitation $o_{\mathbf{q}}$ for the direction $\mathbf{q} \propto(1,0)$.

c): $\delta=0.15$.

d): $\delta=0.2$.

FIG. 12. $\ln |g|$ and $\ln |f|$ as a functions of the distance between sites $r=\sqrt{m^{2}+n^{2}}$. If $m+n$ is odd, the $\ln |g|$ is plotted. And if $m+n$ is even, the $\ln |f|$ is plotted. The curves correspond to hole concentrations $\delta=0.05,0.1,0.15,0.2$.

FIG. 13. Static magnetic formfactor $S_{M}(\mathbf{q})$ at hole concentration $\delta=0.15$. The arguments are $q_{x} / \pi$ and $q_{y} / \pi$. The dashed contour corresponds to the half width. 


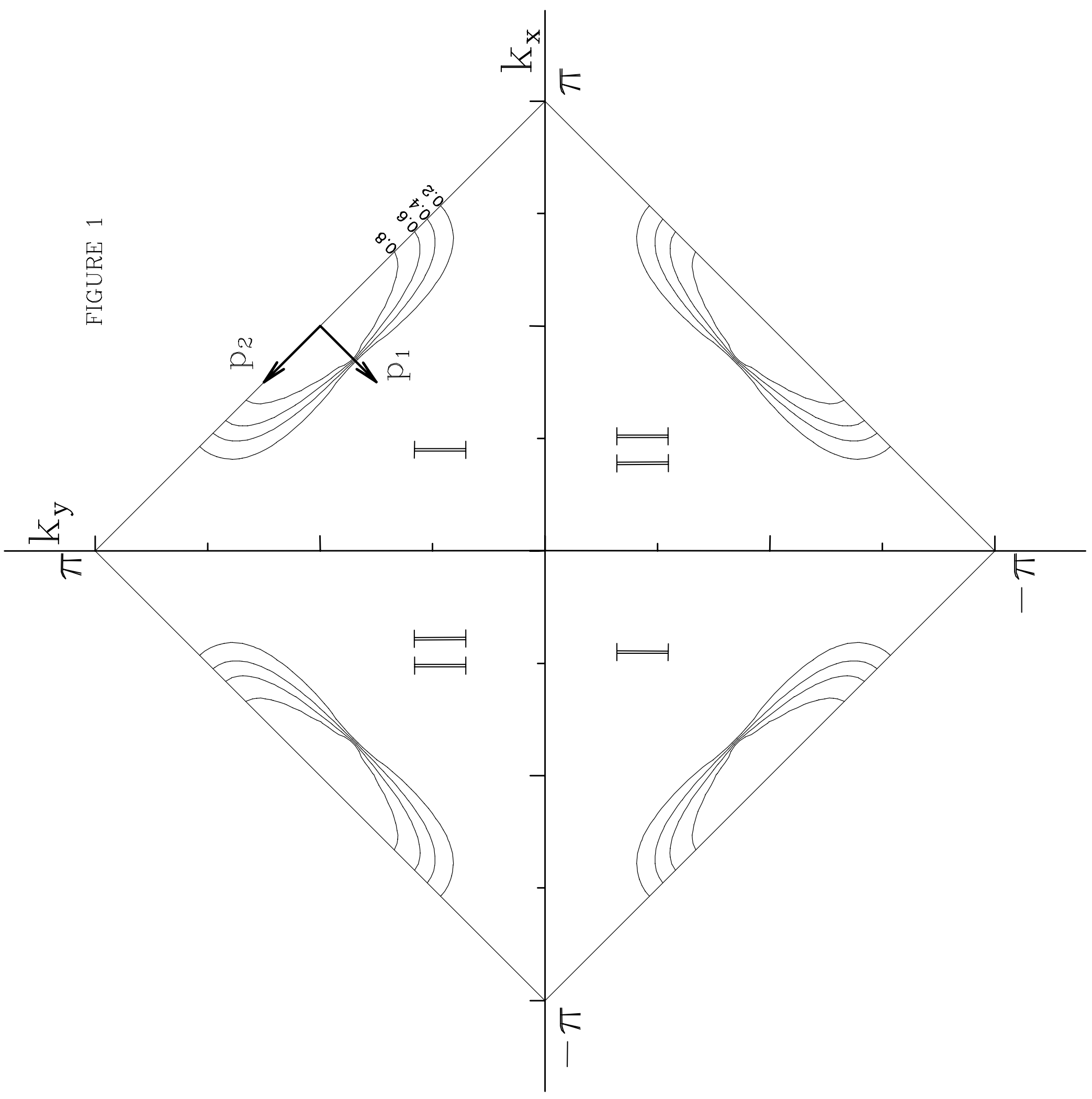



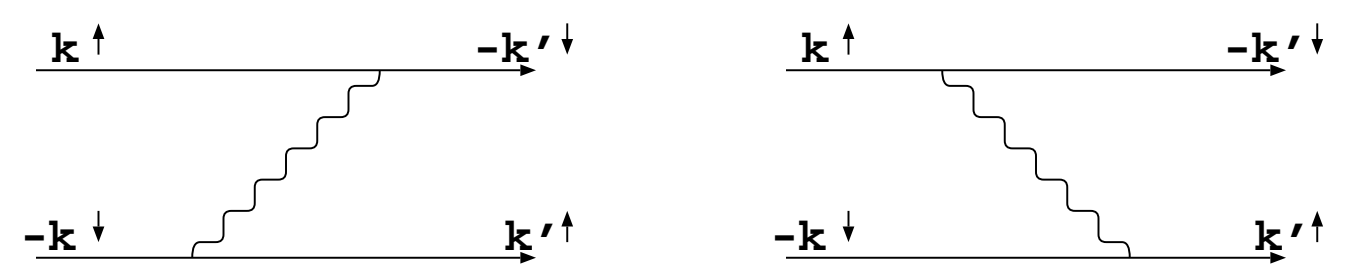

Fig. 2

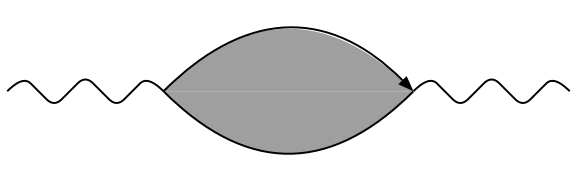

a

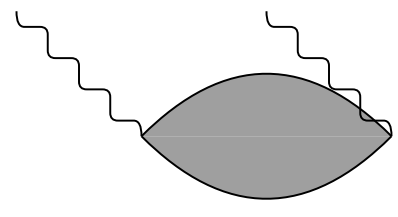

b

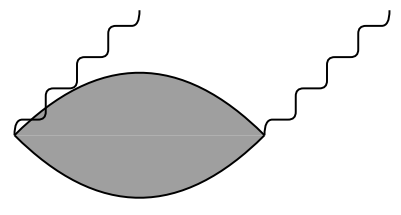

C

Fig. 3

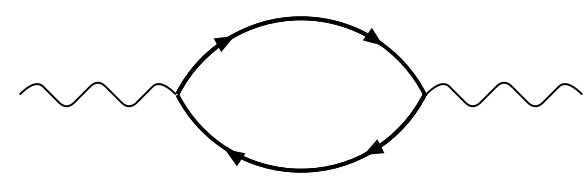

a

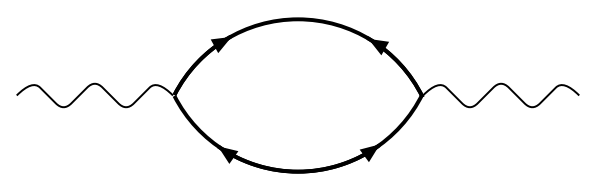

b

Fig. 4

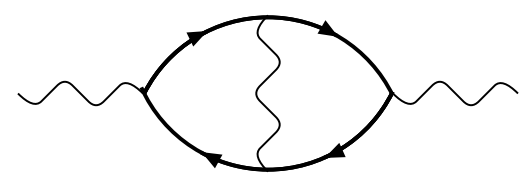

a

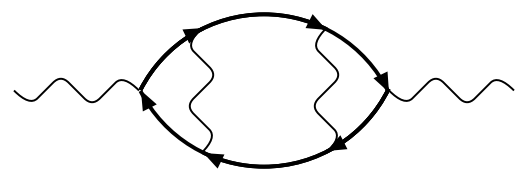

b

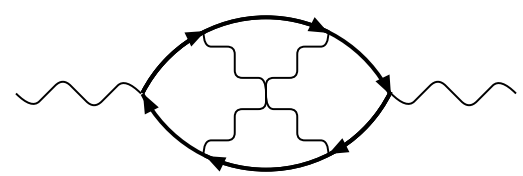

C

Fig. 5 


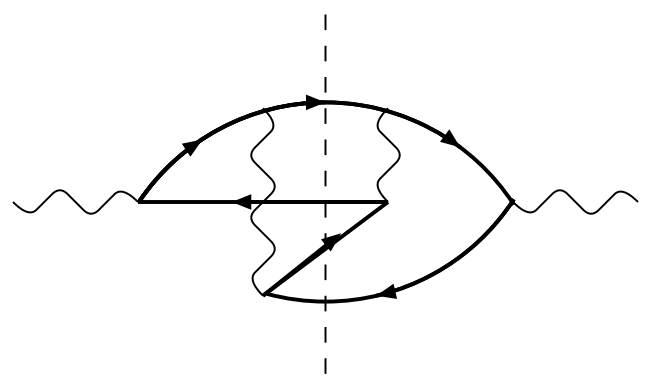

a

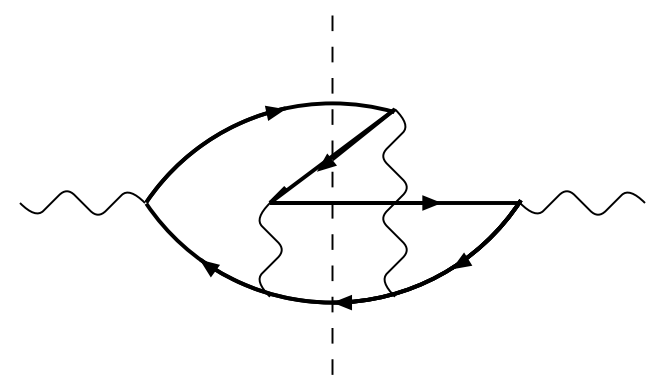

b

Fig. 6

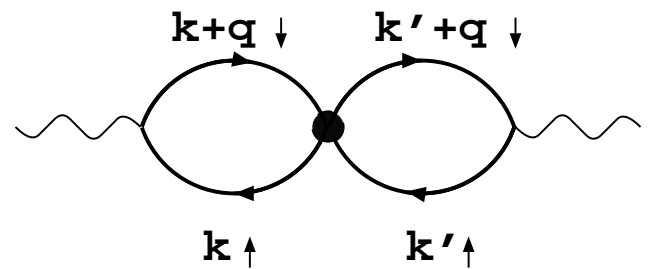

Fig. 7
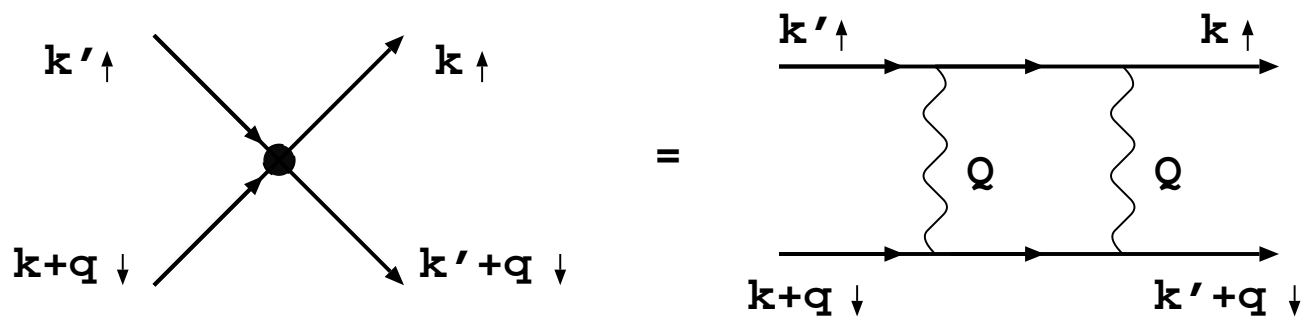

Fig. 8 


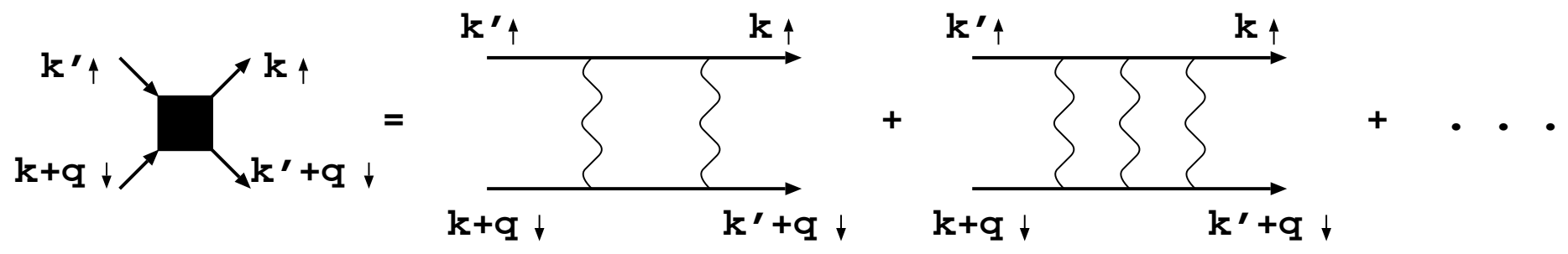

Fig. 9

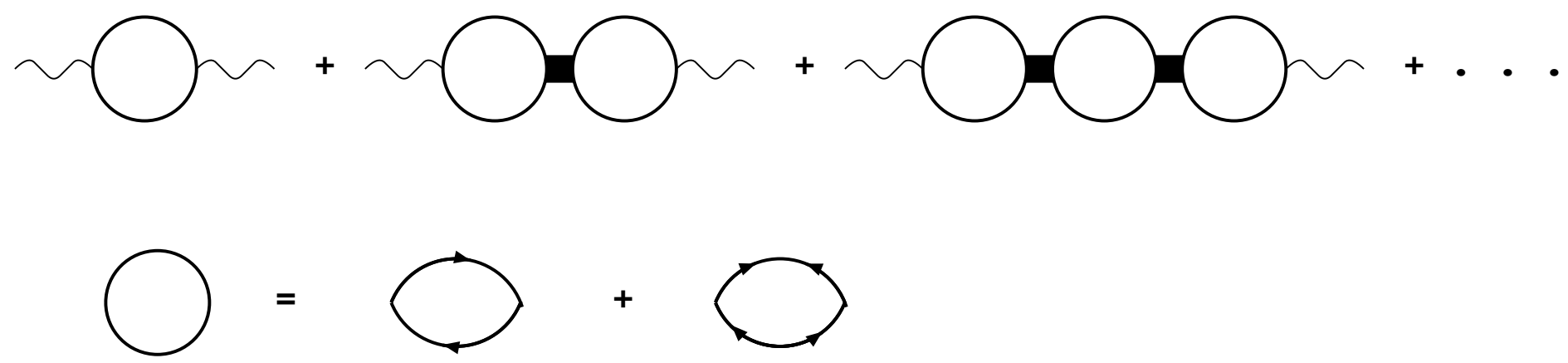

Fig. 10 
Fig. 11a

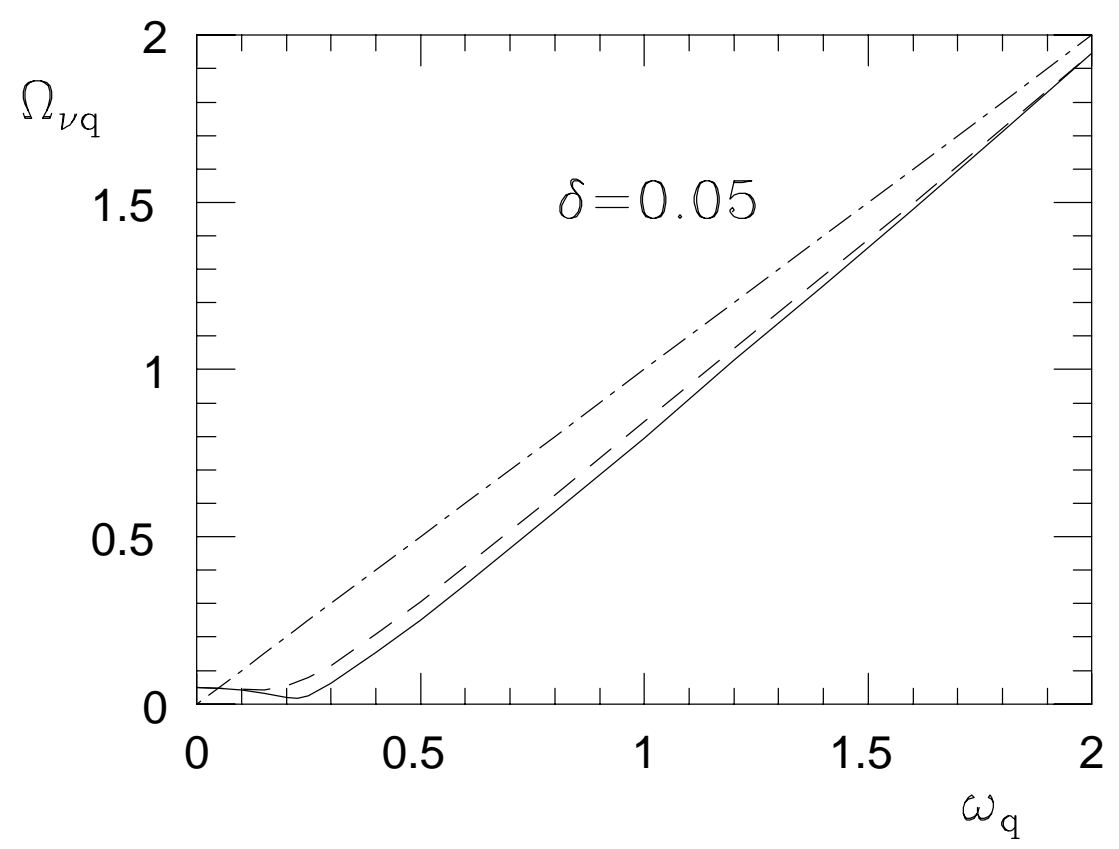

Fig. 11c

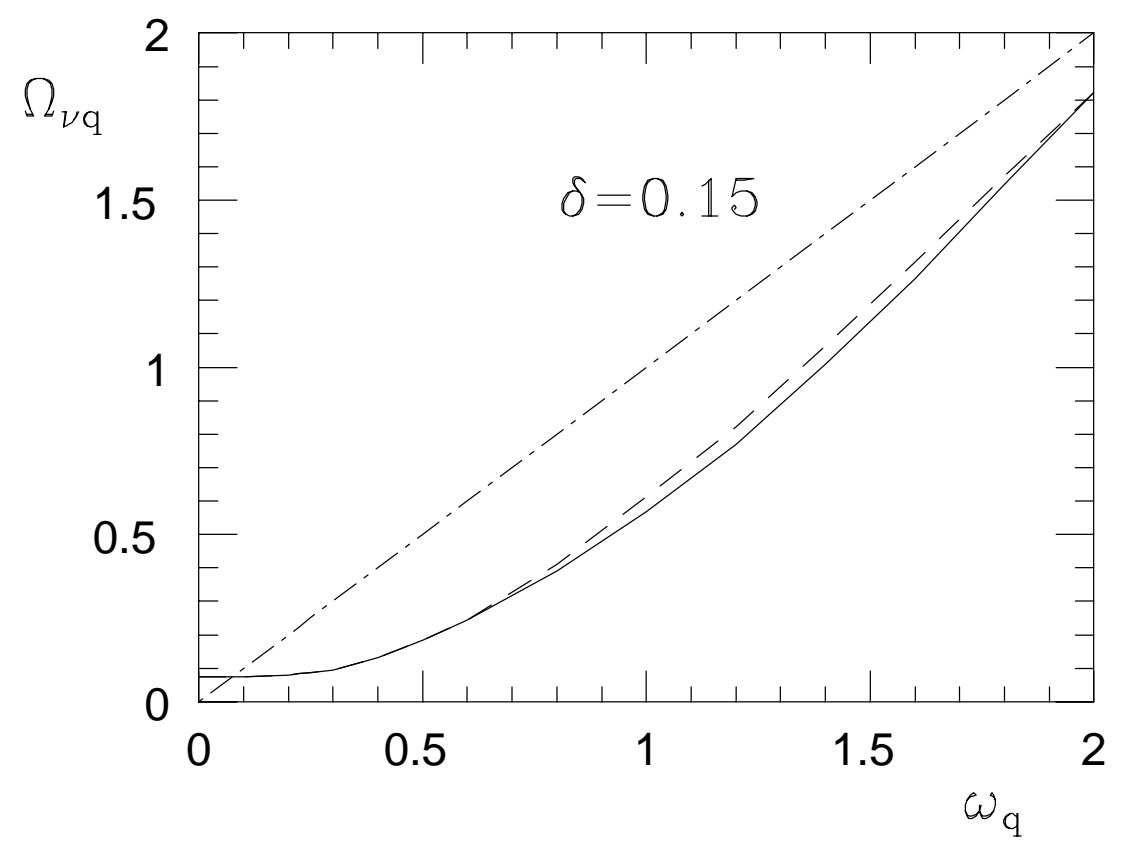

Fig. 11b

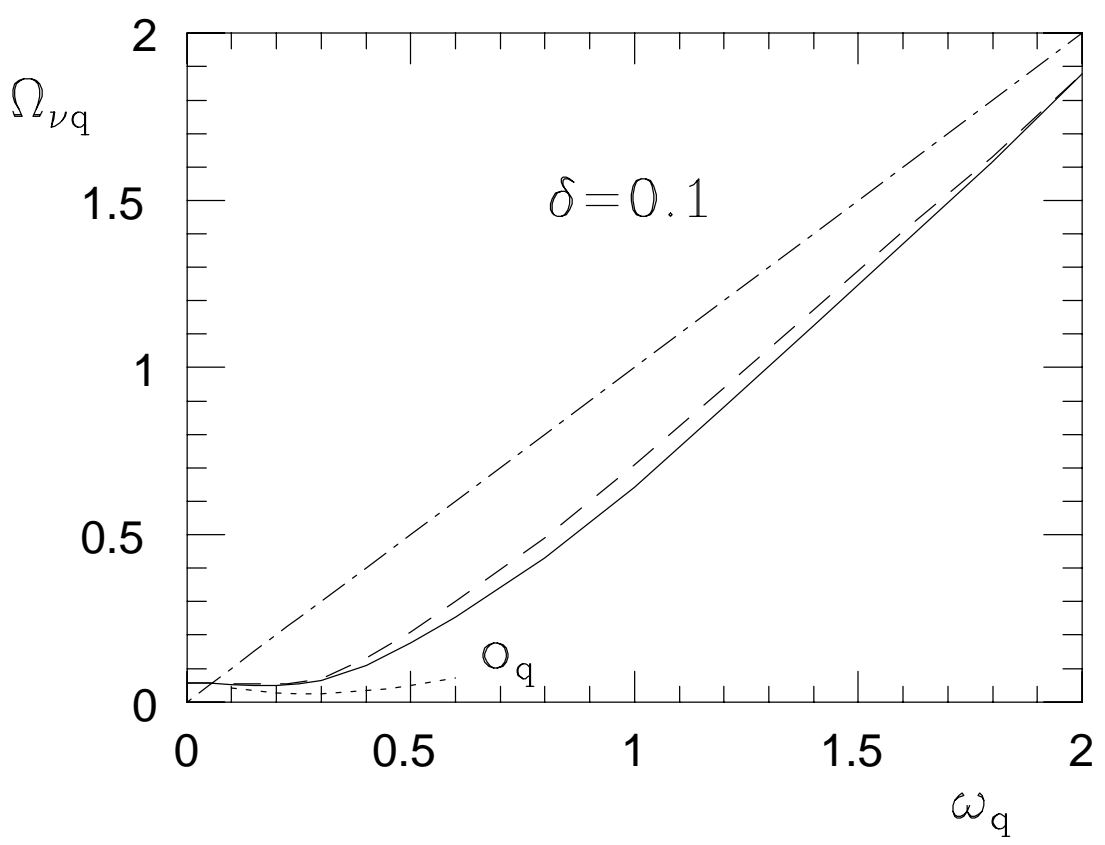

Fig. 11d

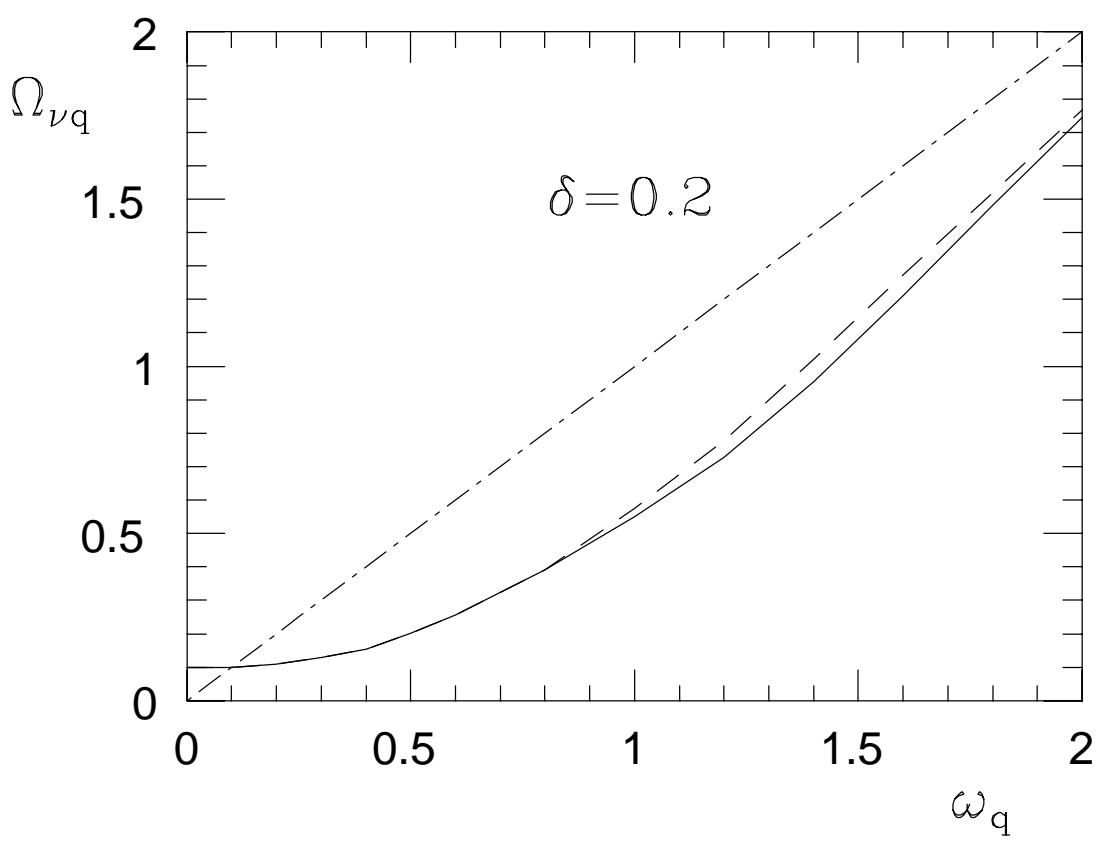




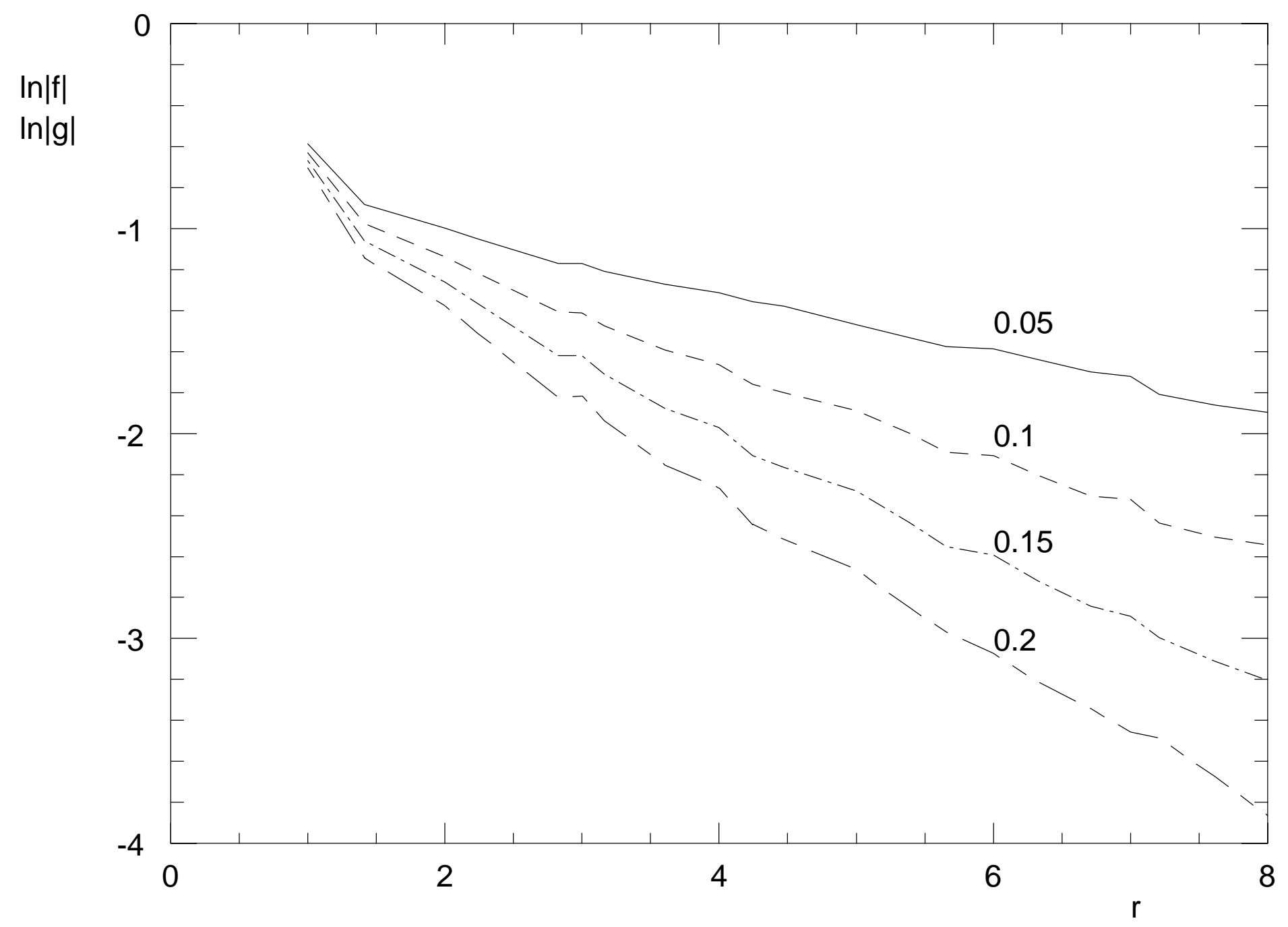

Fig. 12 
Fig.13

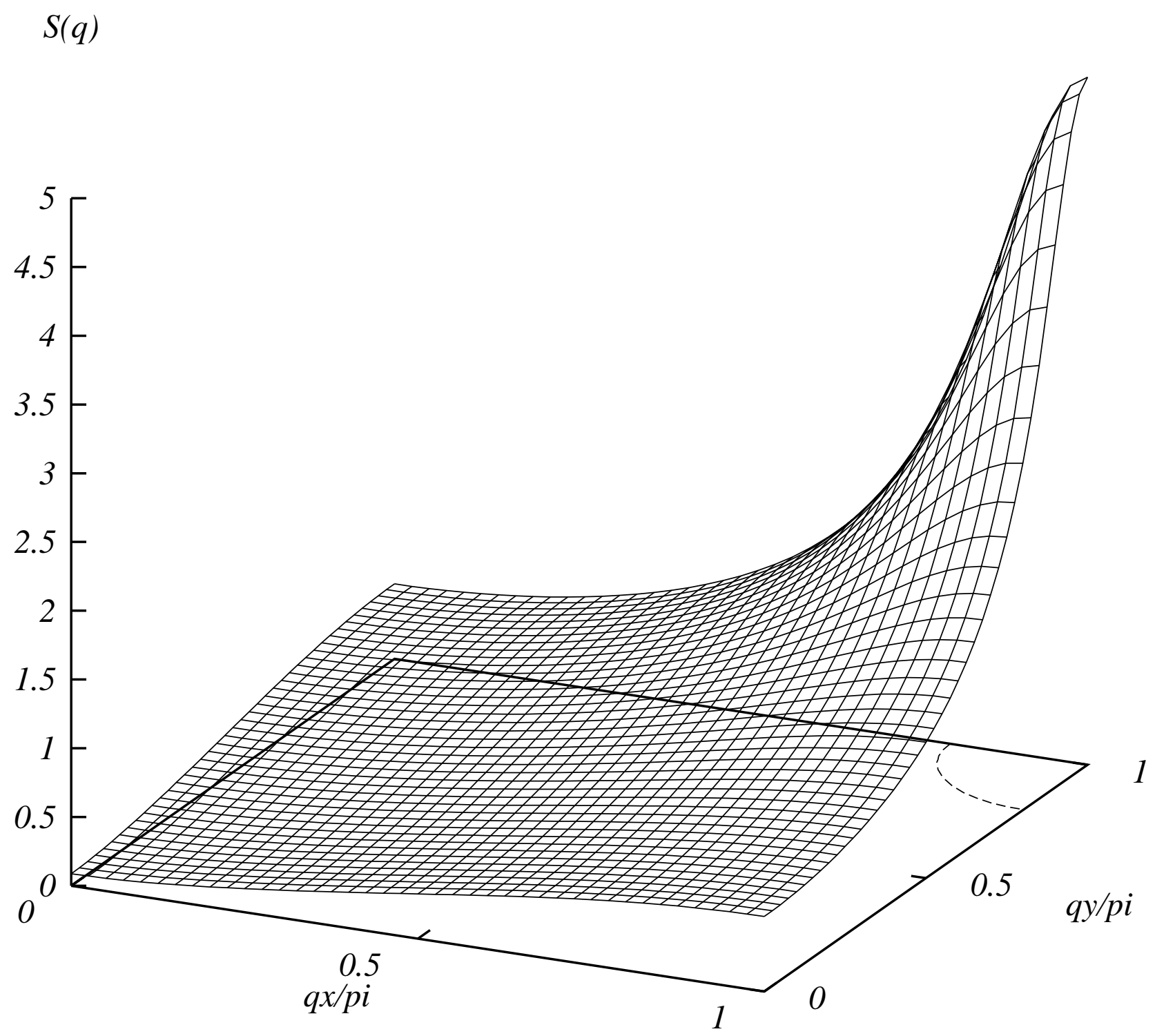

